\title{
Fluidez del mercado laboral y resultados en materia de empleo en Colombia: evidencia derivada de datos enlazados de empleadores y empleados ${ }^{1}$
}

\author{
Leonardo Fabio Morales y Daniel Medina
}

\section{Resumen}

La literatura sobre la flexibilidad de los mercados laborales también se relaciona con el concepto de fluidez entendida en un sentido amplio como la movilidad de trabajadores y empleos en el mercado laboral. Calculamos las mediciones estándar de fluidez del mercado laboral urbano de Colombia y el aumento de la fluidez, especialmente después de 2010. Los modelos de desempleo de equilibrio predicen, en general, una relación negativa entre algunas de las mediciones de la fluidez y la tasa de desempleo de equilibrio. La literatura reciente sobre flujos de trabajadores y empleos señala aspectos benignos de la fluidez, dado que se espera que en los mercados laborales fluidos la duración del desempleo sea en promedio más corta. Encontramos evidencias de un efecto positivo de la fluidez sobre los diferentes índices de empleo y ocupación, utilizando modelos de regresión de variables instrumentales que explotan las variaciones de resultados laborales y la fluidez entre mercados laborales y en el tiempo.

\section{Palabras clave}

Empleo, mercado de trabajo, recursos humanos, movilidad de la mano de obra, medición, estadísticas del empleo, Colombia

\section{Clasificación JEL}

J60, J63, J11

\section{Autores}

Leonardo Fabio Morales es investigador en el Grupo de Análisis de Mercado Laboral en el Banco de la República, Colombia. Correo electrónico: Imoralzu@banrep.gov.co.

Daniel Medina es profesor en formación en el Departamento de Economía de la Universidad EAFIT, Colombia. Correo electrónico: dmedin17@eafit.edu.co.

Los autores agradecen los comentarios de Christian Posso, Carlos Medina, Lina Cardona y a los miembros del Grupo de Análisis de Mercado Laboral del Banco de La República de Colombia (http://investiga.banrep.gov.co/es/grupos-deinvestigacion/grupo-de-analisis-del-mercado-laboral-gamla). En Morales y Medina (2016) figura una versión en inglés de este articulo como documento de trabajo. 


\section{Introducción}

En la literatura reciente sobre la economía laboral se ha focalizado la atención en el grado de dinamismo de los mercados de trabajo de los países desarrollados. A este respecto, se ha documentado abundantemente la pérdida de dinamismo que ha experimentado el mercado laboral de los Estados Unidos en las últimas tres décadas (Bjelland y otros, 2011; Molloy y otros, 2016; Davis y Haltiwanger, 2014; Hyatt y Spletzer, 2013). El concepto de fluidez se ha utilizado en la literatura especializada para señalar la capacidad de una economía de movilizar a los trabajadores y los empleos entre las diferentes empresas, sectores económicos y mercados laborales. La fluidez ha pasado a ser un tema importante en el estudio del mercado laboral, ya que existen pruebas de las consecuencias adversas de una reducción del dinamismo (Davis y Haltiwanger, 2014; Molloy y otros, 2016). Pese a la importancia de este tema, muy poco se sabe acerca de la fluidez del mercado de trabajo en las economías en desarrollo, así como acerca de sus consecuencias respecto al comportamiento de los mercados laborales.

En este estudio calculamos las tasas de reasignación de trabajadores, las tasas de reasignación de empleos y las tasas de rotación o churning, que son las mediciones de fluidez estándar más utilizadas en la literatura. Estas tres tasas son mediciones relativas de lo que la literatura referente a la fluidez del mercado laboral llama flujos de trabajadores, de empleos y de rotación, respectivamente. Por flujo de trabajadores se entiende los movimientos de trabajadores entre las diferentes empresas y sectores económicos, y se mide agregando las contrataciones y desvinculaciones de las empresas. Por flujos de empleos se entiende los movimientos de empleos: se mide agregando el valor absoluto de los cambios netos en las nóminas de las empresas. Por último, por flujos de rotación se entiende el exceso de flujos de trabajadores por sobre el flujo de puestos de trabajo. Utilizamos datos del mercado de trabajo formal de Colombia, una economía en desarrollo de ingresos medios, correspondientes al período transcurrido entre octubre de 2008 y diciembre de 2014. Agregamos estas mediciones para las 23 principales áreas metropolitanas de Colombia. Hemos adoptado una definición amplia de empresa: en este artículo, una empresa es un negocio que cuenta con un local físico o más en una ciudad, con un total de al menos dos empleados. Debe tratarse de establecimientos formales, en el sentido de que efectúan pagos de impuestos a la nómina y aportes patronales de seguridad social correspondientes a sus empleados.

Uno de los puntos fuertes de este artículo es el hecho de que utilizamos un conjunto de datos generados a partir de los registros administrativos del Sistema de Seguridad Social Integral de Colombia. A partir de esos registros, reunimos datos longitudinales correspondientes a empleadores y empleados de todas las empresas formales del país, lo que nos permite calcular las mediciones de la fluidez de todos los sectores económicos. La mayoría de los artículos en la literatura referente a los flujos de empleos y de trabajadores utilizan datos a nivel de establecimientos, que en el mejor de los casos sirven de muestra para sectores económicos específicos, por lo general manufactureros. En algunos casos, en los estudios se hace referencia a todas las empresas formales de todos los sectores, pero la información se limita a una zona geográfica específica de un país, por ejemplo a un determinado estado de los Estados Unidos (Burgess, Lane y Stevens, 2000). La mayor parte de lo que podemos saber a partir de la literatura referente a los flujos de trabajadores y empleos corresponde al sector manufacturero, utilizando paneles rotatorios (Davis, Haltiwanger y Schuh, 1996)².

\footnotetext{
2 Como ejemplos de estas fuentes de información cabe mencionar la Base de Datos de Investigación Longitudinal (Davis, Haltiwanger y Schuh, 1996) y la Encuesta sobre Rotación de la Mano de Obra y Empleos Vacantes (Davis y Haltiwanger, 2014). En el caso de los paneles rotatorios, deben hacerse correcciones a fin de no sobreestimar las mediciones de la fluidez; nuestra medición de la fluidez es válida independientemente de toda corrección porque observamos el universo completo de empresas formales.
} 
El efecto de la fluidez sobre el comportamiento del mercado laboral puede ser positivo si su aumento va acompañado de una reducción en el costo de transición en el mercado, lo que es probable que afecte positivamente a la productividad agregada de la economía (Molloy y otros, 2016). Además, existen razones empíricas y teóricas para considerar que el efecto de la liquidez sobre el comportamiento del mercado laboral es favorable. En primer lugar, según los estudios empíricos la resignación de trabajadores en diferentes empresas y sectores es procíclica, especialmente cuando ella excede a la reasignación de empleos (rotación o churning) (Akerlof y otros, 1988; Davis, Haltiwanger y Schuh, 1996). En segundo lugar, los modelos de desempleo de equilibrio (search models of equilibrium unemployment) - que permiten analizar las transiciones de un empleo a otro- predicen que los factores que reducen el costo de la búsqueda y, por ende, aumentan la reasignación de trabajadores darán lugar a una reducción de la tasa de desempleo de equilibrio.

Entre otras cosas, presentamos evidencias de que el mercado de trabajo formal en Colombia adquirió mayor fluidez después del último trimestre de 2010, coincidiendo con una caída sustancial de la tasa de desempleo ${ }^{3}$. Además, estimamos modelos econométricos que capturan la relación causal entre las mediciones de la fluidez y algunos resultados en materia del mercado laboral. Los resultados que analizamos en este artículo son los del mercado de trabajo formal y asalariado, principalmente porque las mediciones de la fluidez que podemos generar a partir de nuestros datos se refieren exclusivamente a los trabajadores y empleos formales asalariados. En concreto, las variables dependientes de los modelos en este artículo son tasas de empleo formal (población con empleo formal asalariado como proporción de participantes en el mercado de trabajo) y tasas de ocupación formal (población con empleo formal asalariado como proporción de la población en edad de trabajar).

Consideramos que las tasas de reasignación de trabajadores, las tasas de reasignación de empleos y las tasas de rotación tienen un efecto benigno e importante en varios aspectos del comportamiento del mercado laboral. En general, presentamos evidencias de que el incremento de la fluidez del mercado de trabajo da lugar a un crecimiento del empleo formal y de los niveles de ocupación formal de la población en edad de trabajar. Davis y Haltiwanger (2014) llegan a una conclusión similar respecto al mercado laboral de los Estados Unidos; sin embargo, no tenemos constancia de que algún otro estudio presente una evaluación empírica de la hipótesis de que la fluidez beneficia a los mercados de trabajo formales de las economías en desarrollo. En Colombia, al igual que en muchos otros países en desarrollo, la informalidad constituye un grave problema, pero de cualquier forma el mercado de trabajo formal es grande y significativo. En las 23 ciudades más grandes de Colombia, en 2016 casi el 51\% de todos los empleados eran empleados formales; en la región de América Latina en su conjunto, el mercado formal representaba el 54\% del total en 2015. Por lo tanto, para las economías en desarrollo es también fundamental entender mejor los efectos causales de la fluidez sobre el comportamiento del mercado de trabajo formal, en especial porque en numerosos países en desarrollo existe un debate sobre las consecuencias no deseables de políticas económicas que hacen a los mercados más rígidos y escleróticos ${ }^{4}$.

La próxima sección de este artículo comienza describiendo las mediciones de la fluidez utilizadas, lo que es fundamental para entender el resto de este trabajo. En la tercera sección se hace una reseña empírica y teórica de la literatura referente a la relación entre la dinámica del trabajador y del empleo y el comportamiento del mercado laboral. En la cuarta sección se describen las fuentes de nuestros datos, mientras que en la quinta se formulan comentarios sobre la dinámica reciente de las mediciones de la fluidez en Colombia. En la sexta sección se presenta el modelo empírico y en la séptima figuran los resultados de las estimaciones. En la última sección se concluye y se indican las implicancias de política generales.

\footnotetext{
3 Esta evidencia se ha corroborado en análisis recientes en Colombia como el de Morales y Lobo (2017).

4 En Blanchard y Portugal (2001) se describe a los mercados laborales escleróticos como rígidos y estáticos; presentan bajos índices de entrada y salida de la situación de desempleo, así como una elevada duración media del desempleo.
} 


\section{Mediciones de la fluidez}

A efectos de calcular los flujos de trabajadores y empleos, un empleo se define como un cargo que ocupa un trabajador (Davis, Haltiwanger y Schuh 1996). Por ende, todas nuestras mediciones se basan en observaciones acerca del tamaño de una empresa y el flujo de trabajadores que entran y salen de ella. Medimos esos flujos mensualmente respecto de todas las empresas definidas como formales en Colombia entre octubre de 2008 y diciembre de $2014^{5}$. Una empresa $j_{t}$ es un conjunto de establecimientos empresariales ubicados en la misma ciudad y que tienen al menos dos empleados, y la empresa pertenece al sector formal dado que paga impuestos de nómina. Un individuo $i_{j t}$ es un empleado de nómina de la empresa $j$ en el período $t$.

Los registros administrativos se utilizan para generar un conjunto de datos longitudinales vinculados referentes a empleadores y empleados, observando mensualmente la nómina de una determinada empresa. Esta estructura de datos se utiliza para calcular las contrataciones $\left(h_{j t}=\left\{i: i_{t} \in j_{t}\right.\right.$ y $\left.\left.i_{t} \notin j_{t-1}\right\}\right)$ como el conjunto de empleados que se observan en un determinado período pero que no se había observado antes. De igual forma, las desvinculaciones $\left(s_{j t}=\left\{i: i_{t} \notin j_{t}\right.\right.$ y $\left.\left.i_{t} \in j_{t-1}\right\}\right)$ se calculan respecto a los empleados que se observaron en el período anterior pero no se observan en el período actual. Por último, los empleados que siguen en actividad $\left(k_{j t}=\left\{i: i_{t} \in j_{t}\right.\right.$ y $\left.\left.i_{t} \in j_{t-1}\right\}\right)$ son los empleados observados en ambos períodos.

La nómina de la empresa en un momento determinado aparece representada como $e_{j t}=k_{j t}+h_{j t}$. El número de empleos creados y destruidos se calcula a partir de los cambios en la nómina de un período al siguiente. Por lo tanto, la creación de empleo $c_{j t}$ y la destrucción de empleos $d_{j t}$ en la empresa $j$ en el período $t$ se representan como $c_{j t}=1_{\left\{\Delta e_{j t}>0\right\}} \Delta e_{j t}$ y $d_{j t}=-1_{\left\{\Delta e_{j t}<0\right\}} \Delta e_{j t}$, respectivamente. Se suma todo este conjunto de datos para generar mediciones agregadas de la fluidez en el mercado laboral $\mathrm{A}$ (un área metropolitana). Por ende, los flujos agregados de contrataciones $\left(H_{A, t}\right)$, desvinculaciones $\left(S_{A, t}\right)$, creación de empleo $\left(C_{A, t}\right)$ y destrucción de empleo $\left(D_{A, t}\right)$ en el mercado laboral A puede representarse como $H_{A, t}=\sum_{j \in A} h_{j t}, S_{A, t}=\sum_{j \in A} s_{j t}, C_{A, t}=\sum_{j \in A} c_{j t}$ y $D_{A, t}=\sum_{j \in A} d_{j t}$, respectivamente 6 .

Seguimos la literatura previa sobre el tema (Davis, Haltiwanger y Schuh, 1996; Davis y Haltiwanger, 1992), en la que se define el tamaño de la empresa como el promedio móvil de orden 2 de empleo de la empresa, representado por la siguiente ecuación: $x_{j t}=\left(e_{j t}+e_{j t-1}\right) / 2$. El tamaño total de la empresa en un área metropolitana se define como $X_{A, t}=\sum_{j \in A} x_{j t}$. Expresamos los flujos de trabajadores (contrataciones y desvinculaciones) y flujos de empleos (creación y destrucción de empleo) como la tasa total de empleo en el mercado laboral $X_{A, t}$. Por ende, las mediciones de la fluidez utilizadas en este artículo se definen de la siguiente manera:

Tasa de reasignación de trabajadores ${ }^{7}$ (WR) $\left[W R_{A, t}=\left(H_{A, t}+S_{A, t}\right) / X_{A, t}\right]$. Se trata de la suma de las tasas mensuales de contrataciones y desvinculaciones. La reasignación de trabajadores refleja el número de personas que cambian -ya sea de empleador o de situación laboral (empleado/ desempleado) - de un período al siguiente (Davis, Haltiwanger y Schuh, 1996). Mide el número de personas que entran a las empresas o salen de ellas.

5 En 2008 se introdujo la Planilla Integrada de Liquidación de Aportes (PILA), de formato electrónico. Sin embargo, se desaconseja el uso de datos correspondientes a sus primeros seis meses de funcionamiento, ya que algunas empresas comenzaron a presentarla entre enero y junio de 2008. Por precaución, la información obtenida de dicho sistema solo se utiliza a partir del trimestre siguiente, cuando ya todas las empresas en funcionamiento presentaban su PILA. Se coteja la robustez de nuestras estimaciones cambiando el primer mes a partir del cual se miden las variables de fluidez; los resultados no cambian significativamente. Esta verificación de la robustez se examina en la nota 23.

$6 H_{A, t}\left(S_{A, t}\right)$ representa a todas las contrataciones (desvinculaciones) en el área metropolitana $A$ en el período $t . C_{A, t}\left(D_{A, t}\right)$ representa todas las ganancias (pérdidas) de empleo en establecimientos nuevos (que cierran) en fase de crecimiento (reducción). $X_{A, t}$ mide el volumen de empleo en el mercado local $A$.

7 En la literatura, a esto se le denomina a veces rotación de trabajadores. 
Tasa de reasignación de empleos (JR) $\left[J R_{A, t}=\left(C_{A, t}+D_{A, t}\right) / X_{A, t}\right]$. Es la suma de las tasas mensuales de creación y destrucción de empleo. La reasignación de empleos es la cantidad de empleos ganados y perdidos de un período a otro (Davis, Haltiwanger y Schuh, 1996). Mide las oportunidades de pasar de empresas en fase de reducción a empresas en fase de expansión.

Tasa de rotación o churning (CR) $\left[C R_{A, t}=\left(H_{A, t}-C_{A, t}+S_{A, t}-D_{A, t}\right) / X_{A, t}\right]$. Es la diferencia entre la tasa de reasignación de trabajadores y la tasa de reasignación de empleos y por lo general se describe como un exceso de flujos de trabajadores por sobre la cantidad necesaria para acomodar los flujos de trabajadores (Davis y Haltiwanger, 2014). La tasa de rotación capta el número de contrataciones que no son puestos de trabajo creados y el número de desvinculaciones que no son puestos de trabajo destruidos. Los empleos de rotación son aquellos resultantes de reemplazar a trabajadores que fueron separados de sus empleos ya sea porque renunciaron o porque fueron despedidos por el hecho de que las empresas buscaban ajustar mejor su personal a las necesidades de la empresa.

\section{Relación entre la fluidez del mercado laboral y sus resultados}

En una sección posterior de este artículo presentamos pruebas de que en los últimos años se ha incrementado la fluidez del mercado laboral en Colombia. En la literatura especializada se han señalado varios aspectos positivos y otros negativos de contar con mercados de trabajo más fluidos. Se ha documentado en varios artículos una relación positiva entre algunas mediciones de la fluidez y el ciclo económico, pero solo respecto de las economías desarrolladas. También existen algunos marcos teóricos estándar que predicen una relación negativa entre ciertas mediciones de la fluidez y la tasa de desempleo de equilibrio. En esta sección exploramos la relación empírica y teórica entre fluidez y desempeño económico, haciendo hincapié en el desempleo de equilibrio.

En la literatura especializada se han indicado los aspectos negativos de la fluidez del mercado laboral, relativos a la reasignación de los puestos de trabajo. Se examinarán algunos de esos aspectos. Los aumentos en la tasa de reasignación de empleos darán lugar a un incremento del desempleo si se deben al hecho de que la destrucción de empleos excede a la creación de empleos. Una reducción de dicha tasa también puede estar asociada con una mayor seguridad laboral y una menor incidencia del desempleo, que por numerosas razones son características positivas de un mercado laboral. Por ejemplo, la pérdida de un empleo puede dar lugar a menores ingresos durante muchos años después del episodio de desempleo (Davis y Haltiwanger, 2014) ${ }^{8}$. Por último, la literatura sobre los flujos del mercado laboral muestra una regularidad empírica: la relación inversa entre el tamaño de la empresa y el ritmo de reasignación de empleos (Davis y Haltiwanger, 1999). A partir de esa evidencia, una reducción en la tasa de reasignación de empleos puede asociarse con una mayor productividad. Esta asociación se explica por el hecho de que una caída de la tasa de reasignación de empleos puede estar impulsada por un crecimiento en el tamaño de las empresas, ya que las empresas grandes presentan menores niveles de reasignación al tiempo que exhiben una productividad más alta.

En la literatura especializada también se han identificado aspectos benignos de la fluidez del mercado laboral. Un incremento de la tasa de reasignación de empleos puede deberse a que se están creando más empleos de los que se están destruyendo. Además, un mercado laboral más fluido reducirá la duración promedio del desempleo, ya que lleva aparejado un aumento en la frecuencia

\footnotetext{
8 En varios estudios se ha evaluado el impacto de un episodio de desempleo sobre una serie de resultados que van desde la salud hasta el bienestar psicológico, habiéndose detectado importantes efectos negativos. Para examinar esta literatura, consúltese Davis y Von Wachter (2011).
} 
de las ofertas de trabajo. Existe la expectativa de una relación directa entre la fluidez y la movilidad laboral, ya que la existencia de mercados más fluidos va acompañada de un mayor potencial de que los empleados cambien de carrera o pasen a ocupar mejores cargos, lo que constituye una característica positiva de los mercados laborales. Con respecto al proceso de localizar empleados idóneos para el empleador, tal vez ello resulte más fácil de hacer es mercados que son más fluidos. Akerlof y otros (1988) sostienen que la movilidad de empleo a empleo es altamente procíclica y mejora la correspondencia entre los trabajadores y los empleos, creando un beneficio adicional para el sistema de protección social gracias a las reducciones del desempleo.

Otro argumento referente a las ventajas de los mercados fluidos es que la fluidez puede ser el resultado de un mercado laboral menos rígido. En varios artículos se ha llegado a la conclusión de que las leyes de protección del empleo reducen la fluidez del mercado laboral (Blanchard y Portugal, 2001; Gómez-Salvador, Messina y Vallanti, 2004; Boeri y Jimeno, 2005; Decker y otros, 2014). Existen amplias pruebas de que un mercado laboral menos rígido produce mejores resultados agregados, como por ejemplo menores tasas de desempleo y una mayor productividad de la economía. Para el caso concreto de la economía estadounidense, Autor, Donohue III y Schwab (2006), y Autor, Kerr y Kugler (2007) presentan evidencia de los efectos adversos de las leyes de protección del empleo y la productividad total de los factores.

Existe un número significativo de fuentes bibliográficas acerca de los flujos del mercado laboral, centrados en datos provenientes del sector manufacturero de los Estados Unidos. En esta literatura empírica se ha puesto en entredicho la capacidad de las opiniones estándar del ciclo económico real para explicar los movimientos de trabajadores y empleos en la economía. Según el marco macroeconómico prevaleciente de los modelos de ciclo económico real, los flujos de trabajadores y empleos deberían ser neutrales en relación con el ciclo económico. Estas predicciones no se ven respaldadas por los datos; por el contrario, lo que se observa en el sector manufacturero de los Estados Unidos puede resumirse a grandes rasgos de la siguiente manera. La tasa de reasignación de empleos presenta una correlación negativa con los cambios netos en el empleo; vale decir, la economía reestructura la organización de los empleos en las recesiones (Davis, Haltiwanger y Schuh, 1996). Sin embargo, según Akerlof y otros (1988), la tasa de reasignación de trabajadores es levemente procíclica. Dado que la parte de la tasa de reasignación de trabajadores que corresponde a la tasa de reasignación de empleos es contracíclica, se deriva que el exceso de reasignación de trabajadores por sobre la reasignación de empleos (la tasa de rotación) es altamente procíclica. Cabe señalar que en la mayor parte de la literatura citada se calculan correlaciones incondicionales simples entre una medición del ciclo económico y la tasa de reasignación de empleos.

Algunos marcos teóricos de reciente elaboración resultan de utilidad para entender la influencia de los flujos de trabajadores y de empleos sobre el desempeño del mercado laboral. A estos efectos, el mejor modelo es el de la teoría del desempleo de equilibrio de Pissarides (Pissarides, 2000). En una versión bastante general del influyente modelo de búsqueda de Pissarides se hace la predicción teórica de que los aumentos de los flujos de trabajadores reducen el desempleo de equilibrio. Un modelo de búsqueda con destrucción endógena de empleos y búsqueda de empleo por una persona ya empleada predice inequívocamente que un aumento en los flujos de trabajadores (transiciones de empleo a empleo) resultantes de las reducciones en los costos de búsqueda de empleo por una persona ya empleada reducirá la destrucción de puestos de trabajo, aumentará la creación de empleo y reducirá el desempleo de equilibrio ${ }^{9}$. Ese efecto se verá acentuado si el aumento en los flujos de trabajadores induce de alguna manera una mayor idoneidad del trabajador para el puesto de trabajo,

\footnotetext{
9 Técnicamente, en un diagrama tradicional que muestre la curva de Beveridge y la línea de creación de empleo en un espacio de vacantes-desempleo, un aumento de la población que busca empleo modifica y hace más pronunciada la inclinación de la línea de creación de empleo, reduciendo así todas las posibles tasas de desempleo de equilibrio independientemente de lo que suceda con la curva de Beveridge. No obstante, en condiciones normales, la curva de Beveridge se desplaza a la izquierda, incrementando la reducción de la tasa de desempleo de equilibrio.
} 
lo que a su vez incrementa la productividad laboral. No existe mucha bibliografía que brinde evidencia respecto a esta conjetura; sin embargo, Akerlof y otros (1988) muestran que los mercados laborales fluidos hacen posible una mejor correspondencia entre los trabajadores y los puestos de trabajo.

En este artículo ponemos a prueba lo que Davis y Haltiwanger (2014) llaman la "hipótesis del mercado fluido". Según esta hipótesis, los mercados laborales fluidos promueven mayores niveles de empleo. El primer estudio en que se evaluó una relación causal entre fluidez y empleo fue el de Shimer (2001). En el artículo se indica que la proporción de jóvenes en la población en edad de trabajar tiene un efecto positivo en el empleo. En el modelo que se propone allí, ello sucede principalmente porque cuanto más grande es la población de jóvenes en la población en edad de trabajar, más económico resulta para las empresas contratar nuevos trabajadores. En esas circunstancias, para las empresas será más redituable crear puestos de trabajo para solicitantes de empleo más jóvenes, impulsando así la creación de puestos de trabajo y reduciendo el desempleo (Shimer, 2001).

Davis y Haltiwanger (2014) proponen un mecanismo adicional en virtud del cual la fluidez puede impulsar el empleo: cuando los mercados laborales son fluidos los trabajadores reciben ofertas de trabajo más frecuentes, lo que a su vez tiene el efecto de acortar los períodos durante los cuales están desocupados. Desde el punto de vista del trabajador, los períodos de desocupación más cortos evitan la pérdida de capital humano, aumentando así los incentivos para trabajar en el futuro. Además, puede que las empresas discriminen contra las personas que han estado sin trabajo durante más tiempo, por lo que períodos de desocupación más cortos deberían reducir los efectos adversos resultantes de esa situación. Los trabajos de investigación sobre los flujos de trabajadores y empleos en los mercados laborales se concentran en gran medida en las economías desarrolladas ${ }^{10}$. Por otra parte, solo recientemente se ha publicado un número limitado de artículos sobre la fluidez del mercado laboral y sus efectos sobre el empleo. Nuestro artículo contribuye a la literatura sobre este tema en al menos dos formas. En primer lugar, es uno de los pocos artículos en detectar los efectos de la fluidez sobre el empleo y la demanda de trabajo, y, que nos conste, no existe otro estudio sobre el efecto de la fluidez sobre los resultados del mercado laboral para una economía en desarrollo. En segundo lugar, nuestros datos presentan la particularidad de que abarcan a todas las empresas formales de la economía colombiana, lo que significa que nuestras conclusiones no se circunscriben al sector manufacturero, como sucede con la mayoría de los estudios sobre los flujos de trabajadores y empleos y la fluidez.

Estudiar las consecuencias de la fluidez para el empleo formal y la demanda de trabajo formal en los países en desarrollo es fundamental en países como Colombia, donde casi la mitad de los empleados tienen empleos formales; identificar las consecuencias de una mayor fluidez del mercado laboral para la creación de empleo formales es un factor clave para entender dichos mercados. Por diversas razones, especialmente la falta de datos, ha habido pocos intentos de entender los flujos de trabajadores y empleos en los países en desarrollo, así como sus consecuencias: es un vacío en la literatura especializada que este artículo pretende llenar.

\section{Los datos}

Los datos utilizados en este artículo provienen de diferentes fuentes. Las mediciones de la fluidez se generan a partir de los registros administrativos de la Planilla Integrada de Liquidación

\footnotetext{
10 Últimamente ha aumentado el interés en este tema, ya que afecta a la región latinoamericana, especialmente en los lugares donde se producen los flujos de empleo. En Pagés, Pierre y Scarpetta (2009) figura la descripción más amplia de los flujos de mercado laboral en la región latinoamericana. Los trabajos anteriores sobre los flujos del mercado laboral en Colombia se han centrado en la creación y destrucción de empleo en el sector manufacturero (Melo y Ballesteros, 2013 y 2014).
} 
de Aportes (PILA) ${ }^{11}$. PILA es una fuente única de información longitudinal que abarca salarios, contribuciones a los fondos de pensión y seguro de salud, algunas características demográficas básicas y algunos datos básicos sobre las empresas de que se trate. Utilizamos esta información para construir un conjunto de datos longitudinales enlazados de empleadores y empleados observados al menos una vez durante el período transcurrido entre octubre de 2008 y diciembre de 2014. Para el mismo período generamos resultados en materia de mercado laboral y variables de control adicionales utilizando la Gran Encuesta Integrada de Hogares $(G E I H)$ de Colombia ${ }^{12}$. En resumen, nuestros datos incluyen mediciones de la fluidez, resultados en materia de mercados laborales y controles correspondientes a las 23 áreas metropolitanas principales de Colombia con carácter mensual desde octubre de 2008 hasta diciembre de 2014.

Este artículo se focaliza en los asalariados que trabajan en empresas formales, definidas como aquellas que efectúan pagos de impuesto a la nómina y contribuciones al sistema de seguridad social. El gráfico 1 representa el empleo asalariado formal de diferentes tipos. La línea continua se refiere al empleo en empresas formales con más de cinco empleados, según lo informado a través de la PILA. Las líneas oscura continua y oscura clara representan el empleo formal y el total de empleados asalariados, con cifras proporcionadas por la $\mathrm{GEIH}^{13}$. Todas las mediciones del empleo muestran que se ha incrementado sustancialmente, en especial desde 2009. Este aumento en el número de empleados ha tenido lugar en un período de notable crecimiento económico en Colombia.

\section{Gráfico 1}

Colombia: empleo formal total, promedios móviles trimestrales y series mensuales ajustadas estacionalmente, 23 áreas metropolitanas más grandes, octubre de 2008 a diciembre de 2014

(En miles de empleados mensuales)

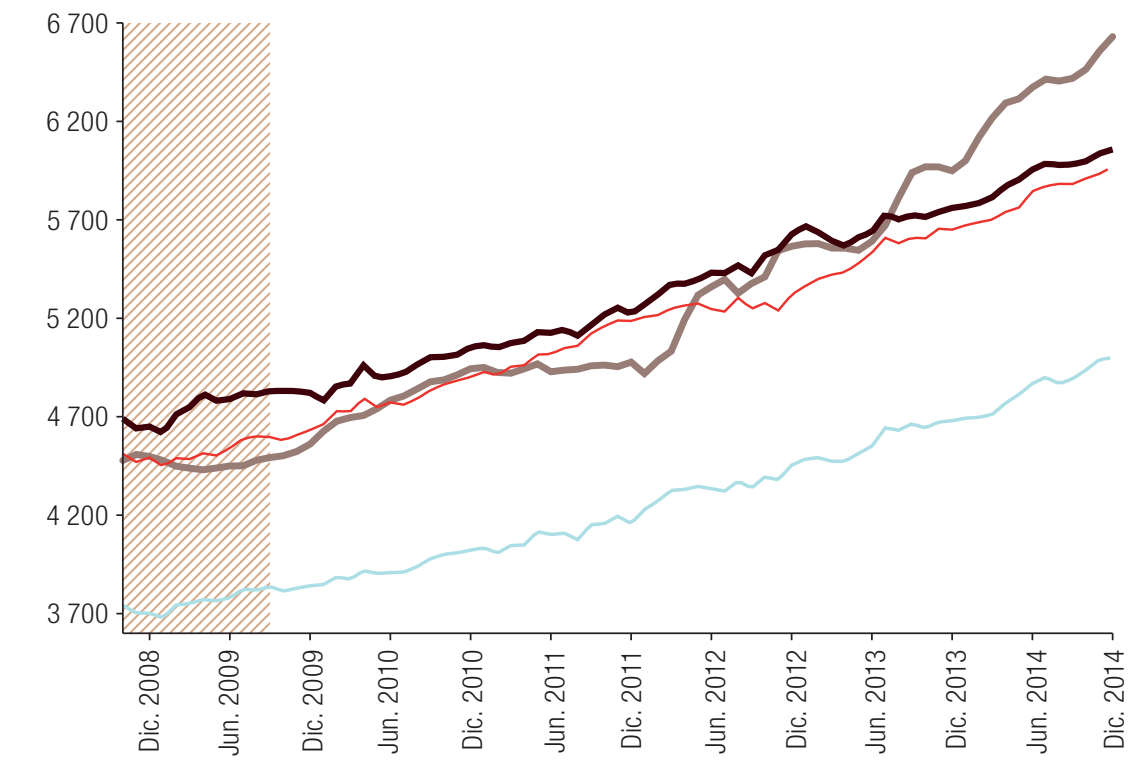

- Empleados — Empleo formal Empleo formal (PILA)

Fuente: Elaboración propia.

a Empresas con más de cinco empleados que presentan su información por medio de la Planilla Integrada de Liquidación de Aportes (PILA), sin imputación de datos.

b Una recesión se define como un período con dos contracciones consecutivas del PIB.

${ }^{11}$ El Ministerio de Salud y Protección Social de Colombia diseñó e implementó un sistema integrado para la recopilación de todos los pagos relativos a la seguridad social que deben hacer los empleadores. Al efectuar esos pagos, los empleadores deben llenar un formulario con información sobre cada empleado que figura en la nómina.

${ }^{12}$ La GEIH se aplica con carácter mensual por el Departamento Administrativo Nacional de Estadística (DANE) de Colombia y es representativa de las 23 áreas metropolitanas más grandes del país.

${ }^{13}$ Según el Departamento Administrativo Nacional de Estadística (DANE) un empleado formal es un empleado asalariado en una empresa con más de cinco empleados o un trabajador profesional por cuenta propia. 


\section{Dinámica reciente de las mediciones de la fluidez en Colombia}

El gráfico 2 muestra los flujos con base en las mediciones tradicionales de la fluidez en la literatura especializada: contrataciones, desvinculaciones, creación de empleo y destrucción de empleo durante el período transcurrido entre octubre de 2008 y diciembre de 2014. El gráfico 2.A presenta flujos en los promedios móviles de orden tres. Lo primero que cabe señalar es que durante el período estudiado en este artículo todos los flujos muestran notables incrementos, especialmente después del último período de recesión económica (junio de 2008 a diciembre de 2009). Los flujos de contrataciones (desvinculaciones) pasó de 410.400 (389.100) trabajadores en el período transcurrido entre enero de 2009 y junio de 2010 a casi 632.800 (594.200) trabajadores en el período entre enero de 2013 y diciembre de 2014. De manera similar, el número de empleos creados (destruidos) se incrementó de 193.100 (201.200) en el período transcurrido entre enero de 2009 y junio de 2010 a 307.000 (333.700) en el período transcurrido entre enero de 2013 y diciembre de 2014. Este período de flujos en crecimiento coincidió con un buen desempeño de la economía colombiana: la tasa de crecimiento anual fue de al menos un $4 \%$ todos los años después de 2009. Antes de eso, el crecimiento económico de Colombia había estado perdiendo dinamismo, y en 2009 el PIB creció solamente un 1,65\%, una cifra reducida en comparación con las tasas subsiguientes.

\section{Gráfico 2}

Colombia: contrataciones, desvinculaciones y creación y destrucción de empleo, promedios móviles trimestrales, 23 áreas metropolitanas más grandes, octubre de 2008 a diciembre de 2014

(En miles de empleados (A) y empleos (B))

A. Empleo formal bruto mensual, contrataciones y desvinculaciones

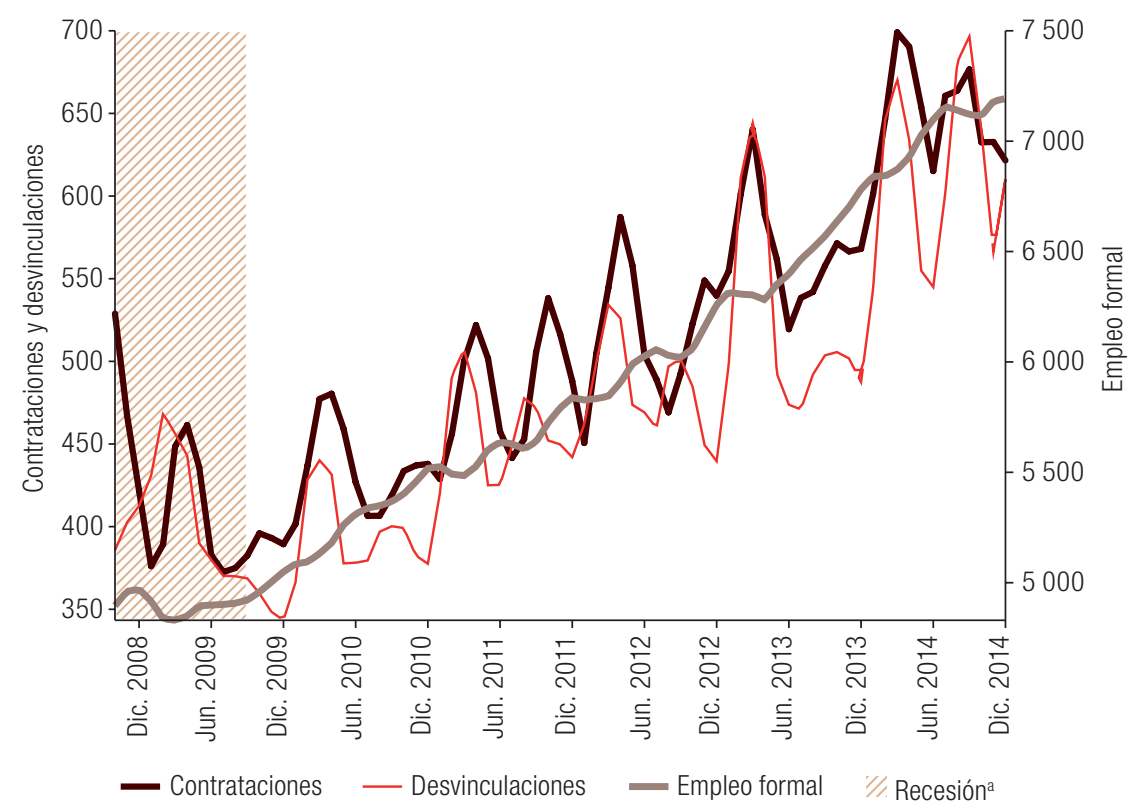


Gráfico 2 (conclusión)

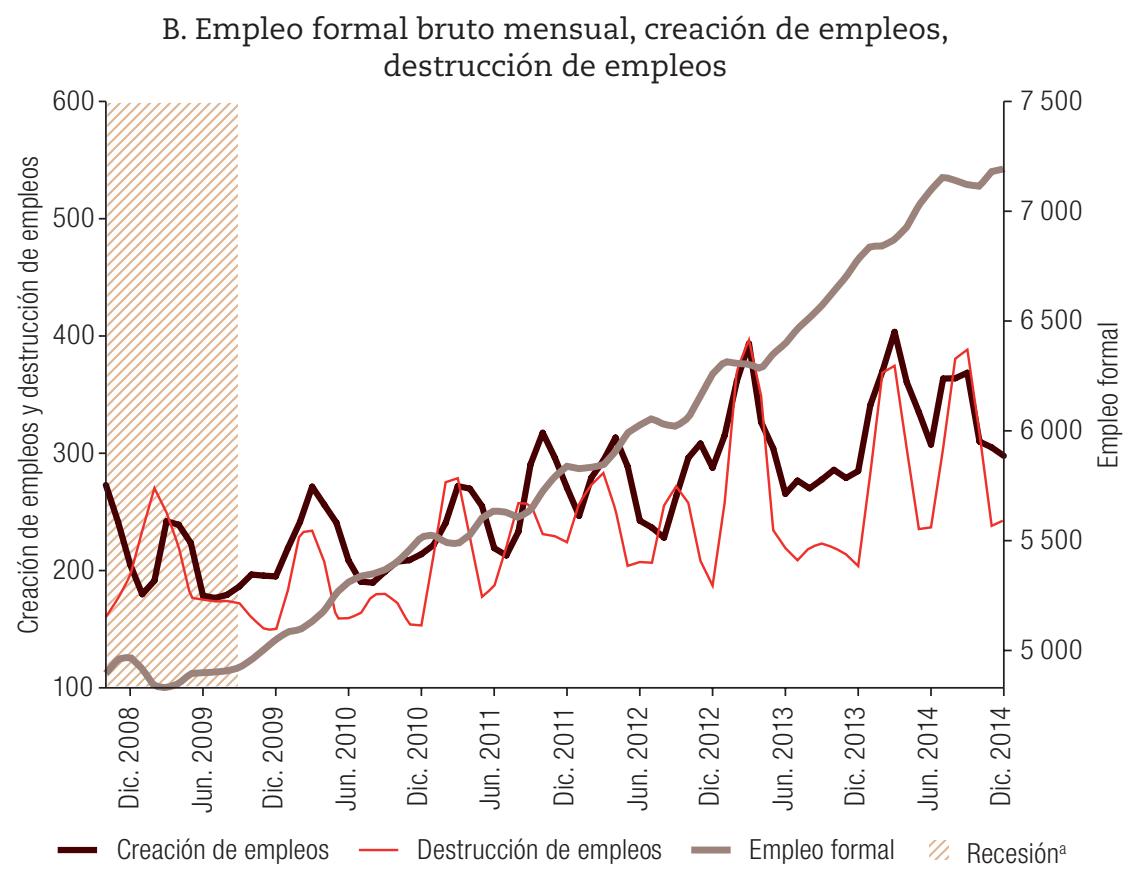

Fuente: Elaboración propia.

a Una recesión se define como un período con dos contracciones consecutivas del PIB.

La relación entre los flujos de trabajadores y de empleos puede caracterizarse a partir de la información que figura en el gráfico 2. Desde octubre de 2008 a diciembre de 2014, el flujo promedio ajustado estacionalmente de trabajadores contratados por todas las empresas formales en Colombia fue de 507.000, mientras que en el mismo período se creó un promedio de 265.000 nuevos puestos de trabajo. Aproximadamente el $52 \%$ de todas las contrataciones eran reemplazos de los trabajadores existentes y no nuevos trabajos. De forma similar, el flujo promedio ajustado estacionalmente de trabajadores separados de las empresas formales en Colombia fue de 477.000, mientras que se destruyeron 234.000 puestos de trabajo. Alrededor del $49 \%$ de las desvinculaciones se debieron a que los trabajadores fueron reemplazados, y no a que se destruyeron puestos de trabajo. Además, cabe señalar que el cambio en el empleo es positivo (negativo) cuando la creación es mayor (menor) que la destrucción. También es cierto que el cambio neto en el empleo ha de equivaler a las contrataciones menos las desvinculaciones, y por ende el cambio en el empleo es positivo (negativo) cuando hay más (menos) contrataciones que desvinculaciones.

El gráfico 3 muestra la relación entre los cambios netos en el empleo y los flujos de contratación y desvinculación, indicando cómo las tasas de creación y destrucción de empleo se relacionan con las tasas de desvinculación y contratación. Este gráfico presenta una representación de la dispersión de las tasas de crecimiento neto del empleo ${ }^{14}$ y las tasas (medianas) de contratación y desvinculación observadas a partir de los datos a nivel del establecimiento; en ella se describe la relación estrecha entre los flujos de trabajadores y el crecimiento del empleo y se muestra que el crecimiento es positivo cuando la tasa de contratación se sitúa por encima de la tasa de desvinculación. Por un lado, las tasas de desvinculación respecto a las empresas en declive son extremadamente elevadas, mientras que las tasas de contratación son bajas y constantes; por otro lado, las tasas de contratación de las empresas en crecimiento son sumamente altas, mientras que las tasas de desvinculación son bajas y constantes.

\footnotetext{
${ }^{14}$ Las tasas de crecimiento neto del empleo se definen como el coeficiente entre los cambios en el empleo y el tamaño de la empresa, definido como $x_{j t}=\left(e_{j t}+e_{j t-1}\right) / 2$.
} 


\section{Gráfico 3}

Colombia: relación transversal entre flujos de trabajadores y crecimiento neto del empleo a nivel de los establecimientos, 23 áreas metropolitanas más grandes, octubre de 2008 a diciembre de 2014

(En porcentajes)

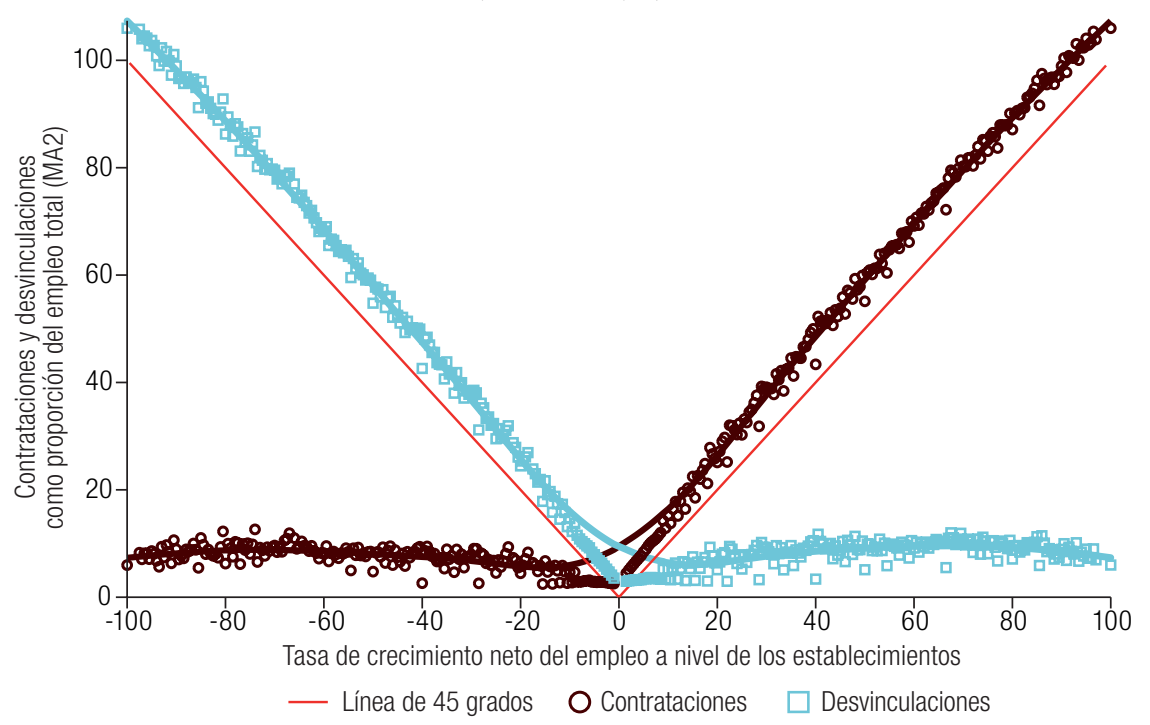

Fuente: Elaboración propia, sobre la base de S. J. Davis, J. Faberman and J. C. Haltiwanger, "Labor market flows in the cross section and over time", Journal of Monetary Economics, vol. 59, No. 1, Amsterdam, Elsevier, 2012.

Nota: Las estimaciones son el promedio ponderado de empleo de las tasas de crecimiento a nivel de los establecimientos dentro de intervalos (0,5 puntos porcentuales). MA2 es un proceso estocástico de promedio móvil con dos desfases temporales.

El comportamiento de las empresas es simétrico en las expansiones y en las contracciones. La relación entre el crecimiento neto y las tasas de contratación o desvinculación muestra lo que en la literatura especializada se conoce habitualmente como formas de palo de hockey. En ambos casos, los pares de tasas de contratación (desvinculación) y las tasas de crecimiento neto se sitúan por encima de la línea de 45 grados. Ello es de esperarse, ya que en las contracciones se observa un nivel de contrataciones pequeño pero no de cero, mientras que en las expansiones se observa un nivel de desvinculaciones limitado pero no de cero ${ }^{15}$. En las expansiones, por ejemplo, a fin de compensar las desvinculaciones la tasa de contratación tiene que ser mayor que la tasa de crecimiento. Como dato interesante, cabe señalar que en las expansiones y contracciones significativas, las líneas correspondientes a las series de contrataciones y desvinculaciones del gráfico 3 se sitúan aún más lejos de la línea de 45 grados. En consecuencia, por ejemplo, hay rápidas expansiones con una tasa de desvinculación notablemente más elevada. Ello tiene sentido porque la rápida expansión hace necesario un mayor nivel de desvinculación para mantener una concordancia adecuada entre empleador y empleado.

El gráfico 4.A muestra flujos de trabajadores y empleos como porcentajes de los niveles de empleo en las áreas metropolitanas. Por su parte, en el gráfico 4.B figuran las tasas de reasignación de trabajadores (WR), las tasas de reasignación de empleos (JR) y la combinación de ambos, la tasa de rotación (CR). El gráfico 4.A muestra que las tasas de contratación y separación se ubicaron entre

\footnotetext{
${ }^{15}$ La relación entre contrataciones, desvinculaciones y tasas de crecimiento del empleo sigue patrones similares en las diferentes economías. Una versión del gráfico 3 presentada por Davis, Faberman and Haltiwanger (2012) respecto a la economía de los Estados Unidos es similar a la que se presenta en este artículo. Ello se debe a la estrecha relación entre los flujos de trabajadores y el crecimiento del empleo; tal como pronosticaría todo modelo económico de demanda de mano de obra, las empresas deberían expandirse a raíz de los incrementos en las contrataciones, y contraerse como resultado del incremento de las desvinculaciones.
} 
el 6,6\% y el $12,0 \%$ durante el período estudiado. La tasa de contratación promedio fue del 8,9\%, mientras que la tasa de desvinculación promedio alcanzó el 8,4\%. Cuando la tasa de contratación se sitúa por encima de la tasa de desvinculación, se observa una gran reducción en los niveles de desempleo (véase el gráfico 4.B).

\section{Gráfico 4}

Colombia: mediciones de la fluidez, promedios móviles trimestrales, 23 áreas metropolitanas más grandes, octubre de 2008 a diciembre de 2014

$$
\text { (En porcentajes de empleo) }
$$

A. Tasas mensuales de contrataciones, desvinculaciones y creación y destrucción de empleo

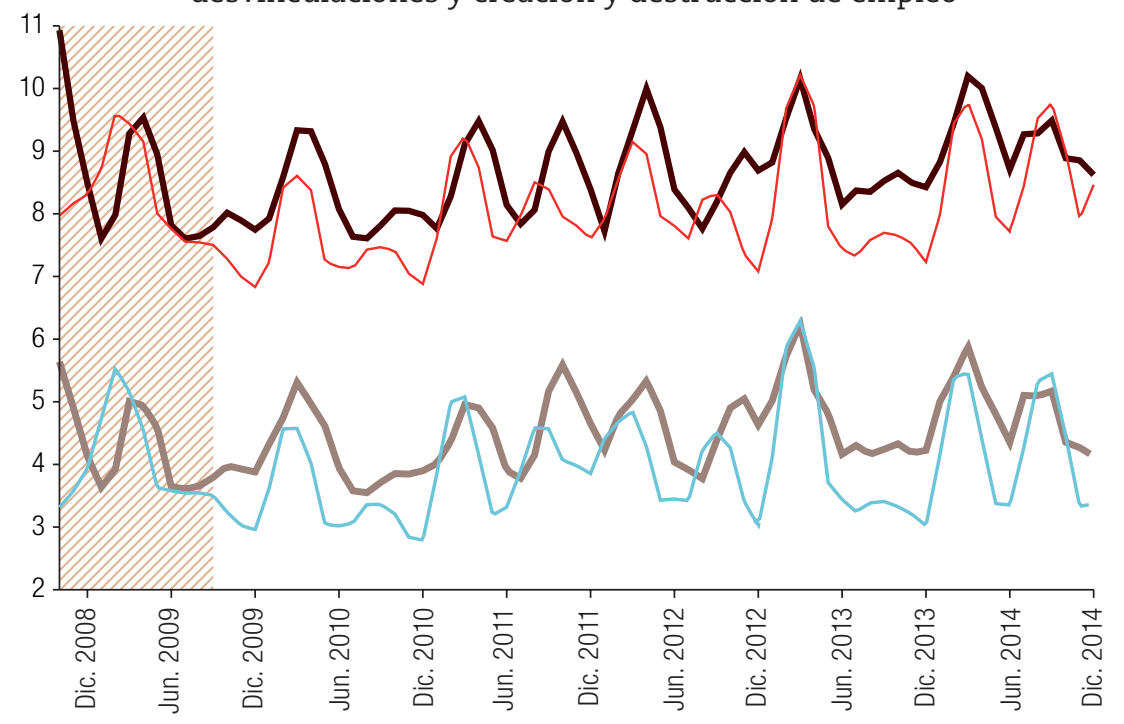

— Contrataciones — Desvinculaciones Creación de empleo — Destrucción de empleo ※//Recesión ${ }^{a}$

B. Tasas mensuales de reasignación de empleos, reasignación de trabajadores, y rotación

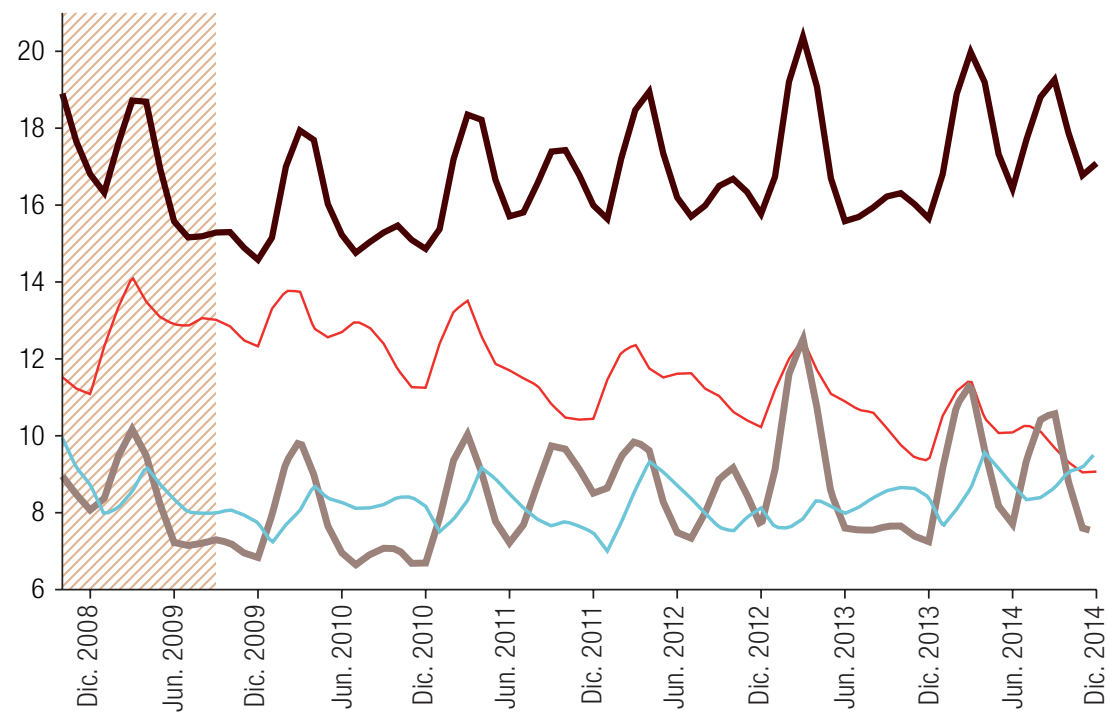

— Reasignación de trabajadores — Tasa de desempleo — Reasignación de empleos — Tasa de rotación " Recesión ${ }^{\mathrm{a}}$

Fuente: Elaboración propia.

a Una recesión se define como un período con dos contracciones consecutivas del PIB. 
Como se mencionó anteriormente, las tasas de creación y destrucción de empleo son tan solo proporciones de las tasas de contratación y desvinculación, respectivamente. En promedio, la tasa de creación de empleo es el $60 \%$ de la tasa de contratación, mientras que la tasa de destrucción es el $57 \%$ de la tasa de desvinculación. Por lo tanto, la destrucción representa un mayor porcentaje del total de desvinculaciones que la creación del total de las contrataciones. El gráfico 4.B muestra que tanto la tasa de reasignación de trabajadores como la tasa de reasignación de empleos presentan un notable incremento en su magnitud y volatilidad después del tercer trimestre de 2010. La combinación de las dos tasas genera la tasa de rotación (churning), que también se incrementó después de 2011.

En el gráfico 4.B se compara la evolución del desempleo y nuestras tres mediciones de la fluidez del mercado laboral, la tasa de rotación, la tasa de reasignación de trabajadores y la tasa de reasignación de empleos. En los tres casos parece haber una relación negativa entre el desempleo y las tres mediciones después de 2009. Cuando el desempleo muestra una tendencia decreciente, las mediciones de la fluidez parecen incrementarse.

En este artículo, a diferencia de lo que ocurre en la mayor parte de la literatura especializada, los flujos laborales se miden respecto de todos los sectores formales de la economía colombiana, no solo respecto de la manufactura. No se pretende aquí caracterizar la heterogeneidad de la fluidez del mercado laboral en los diferentes sectores económicos; sin embargo, se puede hacer mención de parte de la evidencia recogida en Flórez y otros (2018), en que se hace un análisis pormenorizado de la heterogeneidad de los flujos laborales en el mercado formal colombiano. Una de las principales conclusiones de ese estudio ${ }^{16}$ es que la manufactura, el sector más estudiado en la literatura especializada internacional, es uno de los sectores menos dinámicos del mercado laboral: las tasas de reasignación de trabajadores, de reasignación de empleos y de rotación fueron menores que las de los demás sectores durante el período 2009-2016. En general, todos los demás sectores económicos presentan tasas de reasignación bastante similares, pero hay uno que se destaca por sus elevadas tasas, a saber, el de la construcción. Las causas y consecuencias de esta heterogeneidad en la dinámica laboral constituyen un tema que aún no ha sido examinado en la literatura especializada.

\section{Modelo empírico}

El propósito de este artículo es determinar el papel que desempeña la fluidez del mercado laboral en las tasas de empleo y ocupación. La definición de "mercado laboral" es un área metropolitana. En Colombia hay 23 grandes áreas metropolitanas. Calculamos las mediciones de la fluidez estándar y las variables para cada área metropolitana en cada período (mes) desde octubre de 2008 hasta diciembre de 2014. La ecuación que estimamos puede representarse de la siguiente manera:

$$
y_{i t}=x_{i t}{ }^{\prime} \beta+\delta_{i}+\tau_{i, t}+\alpha f_{i t}+\varepsilon_{i t}
$$

donde $y_{i t}$ es el desempeño del mercado laboral (tasas de empleo y ocupación definidas para los trabajadores asalariados formales) en el área metropolitana $i$ en el período $t, \delta_{i}$ es el efecto fijo por área metropolitana, el vector $x_{i t}$ contiene una serie de variables de control que varían por área metropolitana, $\tau_{i t}$ denota una serie de efectos fijos de tiempo. El coeficiente de interés es $\alpha$, que capta cómo cambia el desempeño del mercado de trabajo $y_{i t}$ cuando la medición de la fluidez $f_{i t}$ aumenta

\footnotetext{
${ }^{16}$ Las tasas mensuales de reasignación de trabajadores estimadas para el mercado laboral formal colombiano en Flórez y otros (2017) muestran tasas de reasignación de trabajadores del 11,84\% para el sector manufacturero y del 18\% para el conjunto del mercado laboral durante el período comprendido entre 2009 y 2016 . Durante este mismo período, el sector de la construcción tuvo una tasa de reasignación de trabajadores del 36\%.
} 
1 punto porcentual. Las variables de control que incluimos en el vector $x_{i t}$ son las siguientes: el salario por hora deflactado por el deflactor implícito del PIB; la proporción de la población en edad de trabajar que ha recibido educación terciaria; la proporción de empresas del sector privado; la edad promedio de los empleados, la proporción de hombres en el empleo asalariado; la demanda de mano de obra a nivel departamental17; el PIB por persona empleada; los costos de los empleadores; el filtro HodrickPrescott por PIB trimestral; y el crecimiento anual del PIB del estado.

Existen varias razones por las que $\alpha$ puede estar afectado por un sesgo de endogeneidad. En el área metropolitana puede haber factores no observados que varían con el tiempo y que determinan el desempeño del mercado laboral $y_{i t}$, al tiempo que están correlacionados con la medición de la fluidez $f_{i t}$. Además, dado que elaboramos mediciones de la fluidez a partir de registros administrativos, estas mediciones pueden ser susceptibles de error en la medición; por ejemplo, en algunos períodos las empresas pueden presentar información de nómina incorrecta. Estas imperfecciones en el conjunto de datos longitudinales correspondientes a empleadores y empleados generan errores en la estimación de las mediciones de la fluidez, y puede pasar que el error de medición en las mediciones de la fluidez no sea aleatorio. Por todas esas razones estimamos modelos variables instrumentales.

\section{Variables instrumentales}

A partir de la información que figura en la literatura especializada anterior, construimos instrumentos válidos plausibles para cada una de nuestras variables endógenas. Utilizamos dos tipos de instrumentos. En primer lugar, usamos características del mercado laboral local que, en función de las variables de control incluidas en las regresiones, se da por sentado que son exógenas. En segundo lugar, siguiendo a Davis y Haltiwanger (2014), construimos instrumentos basados en mediciones nacionales de intensidad de la fluidez por sectores económicos; esos instrumentos incorporan los cambios en la fluidez del mercado laboral nacional a la intensidad de la fluidez en las áreas metropolitanas.

Instrumentos basados en las características de los mercados locales: Utilizamos una serie de instrumentos basándonos en la idea de que los trabajadores jóvenes con escasa formación tienen la mayor movilidad en el mercado laboral (Shimer, 2001; Davis y Haltiwanger, 2014). En primer lugar, tomamos la población de entre 18 y 24 años de edad sin educación secundaria o que a lo máximo haya terminado la enseñanza secundaria, en ambos casos como porcentaje de la población en edad de trabajar. En segundo lugar, tomamos el salario mínimo, en el entendido de que necesariamente se aplica a trabajadores jóvenes con escasa formación. Para el uso de estos instrumentos es necesario controlar el salario medio por hora en cada mercado laboral local. El salario mínimo es un factor exógeno que frecuentemente impone al mercado el gobierno ${ }^{18}$. Por otra parte, los trabajadores y las empresas, actuando en forma individual, prácticamente no tienen ninguna oportunidad de ejercer influencia al respecto. Por ende, al controlar el salario promedio por área metropolitana, podemos asumir que el salario mínimo no guarda correlación con el error en la ecuación (1).

Instrumentos basados en la intensidad de la reasignación de los sectores económicos: En este caso seguimos la literatura referente a las mediciones de la demanda local de trabajadores. Bartik (1991) elabora una medición para los cambios en la demanda local de mano de obra que no está relacionada con la oferta local de trabajadores. La idea consiste en incorporar los cambios en el empleo "nacional" a los cambios en el empleo local (por "nacional" entendemos empleo total menos local). Con ese propósito, se hace un promedio de los cambios en el empleo nacional en las

\footnotetext{
17 En la sección VI.1 se explica en detalle cómo se elaboró este control.

${ }^{18}$ En cinco de siete años desde 2008 a 2014, el gobierno impuso el salario mínimo porque la negociación entre los sindicatos y las empresas no dio lugar a ningún acuerdo.
} 
distintas industrias, utilizando los porcentajes de empleo de la industria local como ponderaciones. En el presente artículo utilizamos el índice original de Bartik de cambios en la demanda local de trabajadores como variable de control, que queda construido de la siguiente manera:

$$
\begin{gathered}
B_{a t}=\sum_{k=1}^{K} \gamma_{k a t-1} \cdot \Delta_{k t}^{-} \\
\text {en que } \Delta_{k t}^{-}=\frac{\Delta E_{k t}^{-}}{1 / 2\left(E_{k t}^{-}+E_{k t-1}^{-}\right)}
\end{gathered}
$$

donde $\gamma_{k a t-1}$ es el porcentaje de empleo del mercado local $a$ en el sector económico $k$ y $\Delta_{k t}^{-}$es el cambio en el empleo en el sector económico $k$ en el momento $t, E_{k t}^{-}$, como porcentaje de nuestra medición del tamaño de la empresa (excluido el mercado laboral local a). Esta tasa especial de crecimiento suele utilizarse en la literatura sobre flujos de trabajadores y empleos y se conoce como crecimiento del empleo neto de Davis, Haltiwanger y Schuh (DHS) (Davis, Haltiwanger y Schuh, 1996). La variable $B_{a t}$ predice cuál habría sido el cambio del empleo neto del mercado laboral local $a$, dado el crecimiento del empleo neto en otros mercados laborales, así como su propia composición industrial.

En diversos artículos se han elaborado instrumentos con arreglo a la idea original de Bartik. Al respecto, sirven de ejemplo los artículos de Blanchard y Katz (1992), Bound y Holzer (2000), y Autor y Duggan (2003). Para un estudio específico de la fluidez de los mercados laborales, Haltiwanger y Davis (2014) proponen una serie de instrumentos derivados de la interacción entre los índices de demanda local de trabajadores y las tasas de reasignación de empleos. En este artículo recurrimos al conjunto de la literatura especializada existente para elaborar nuestro instrumento de intensidad de reasignación, a saber,

$$
B I_{a t}=\sum_{k=1}^{K} \gamma_{k a t-1} \cdot f_{k t}^{-}
$$

donde $f_{k t}^{-}$es una medición de la fluidez (WR, JR, CR) para el sector $k$ calculado a nivel nacional, pero se excluye al mercado local $a$. Además, $\gamma_{k a t-1}$ es el porcentaje de empleo del mercado local $a$ en el sector económico $k$ en el período anterior. Este instrumento capta la interacción de las mediciones de la fluidez de las diferentes industrias en otros mercados laborales dada la anterior composición industrial del mercado laboral local. El instrumento $B I_{a t}$ utiliza las mediciones de la fluidez nacional (excluido el nivel local) de diferentes sectores económicos a fin de predecir las mediciones de la fluidez local. En la elaboración de estos instrumentos, las mediciones de la fluidez "nacional" se ponderan por el porcentaje rezagado del empleo de un sector en la composición industrial local.

Comentarios sobre la validez de los instrumentos: Con respecto al primer grupo de instrumentos, damos por sentado que son exógenos, dado el conjunto de controles que utilizamos en la ecuación estructural. Considerando el salario mínimo real, este podría estar correlacionado con el ciclo económico y con otros factores relevantes que afectan el desempeño del mercado, aunque generalmente aquel viene impuesto por el Gobierno. Por ende, en nuestra ecuación estructural incluimos el PIB por persona empleada, un filtro Hodrick-Prescott del PIB trimestral y el crecimiento anual departamental del PIB. Además, en las regresiones controlamos el salario promedio del mercado laboral en promedio móvil. En vista de que tomamos en cuenta el ciclo económico y el efecto de los salarios promedio en el desempeño de los mercados laborales, asumimos que los salarios mínimos no están correlacionados con el término de error en la ecuación (1). En el caso de los porcentajes de la población de entre 18 y 24 años de edad con diferentes niveles de educación, en la literatura especializada por lo general se da por sentado que son exógenas, ya que reciben una marcada 
influencia de las tendencias demográficas; por lo tanto, es poco probable que mantengan una correlación con los ciclos económicos o una heterogeneidad no observada que varía con el tiempo ${ }^{19}$.

Con respecto a los instrumentos del tipo Bartik, incluimos $B_{a t}$ como variable de control en nuestra ecuación estructural; asumimos que los instrumentos $B I_{a t}$ no guardan correlación con el término de error en la ecuación (1), a condición de que esta variable se incluya en la regresión. Por distintos motivos, es posible que así sea. En primer lugar, estos instrumentos aíslan los cambios en las intensidades de reasignación que se derivan de los cambios en la intensidad de la reasignación a nivel de la industria. Se calculan utilizando otras medidas de reasignación del área metropolitana y la composición rezagada del empleo de un área metropolitana en particular (Davis y Haltiwanger, 2014). En segundo lugar, tuvimos en cuenta diversas mediciones del ciclo económico y las predicciones de los cambios en la demanda laboral utilizando cambios en dicha demanda en otras áreas metropolitanas. Dado que tenemos en cuenta $B_{a t}$, nuestros instrumentos del tipo Bartik no guardan correlación con las perturbaciones que varían con el tiempo y que afectan la demanda de mano de obra a nivel de la industria; por ende, muy probablemente, la única forma en que la reasignación a nivel de la industria podría de forma afectar el desempeño de los mercados laborales es a través de su efecto sobre la reasignación local.

En todas nuestras regresiones variables instrumentales utilizamos modelos sobreidentificados, empleando varios de los instrumentos propuestos en los párrafos anteriores. Hacemos uso de la prueba de significación conjunta ( $F$-test) en la primera etapa a fin de poner a prueba la solidez de nuestros instrumentos. Con respecto a la prueba estándar para las restricciones de sobreidentificación, las utilizamos con el objetivo de poner a prueba la validez o exogeneidad de restricciones de exclusión adicionales ${ }^{20}$. En cuanto a la potencia de los instrumentos, los estadísticos $\mathrm{F}$ provenientes de la primera etapa de las regresiones son mayores que 13 en todos los casos, y en ningún caso rechazamos la hipótesis de que el instrumento fuera válido en términos del test de restricciones de identificación.

\section{Resultados}

En esta sección mostramos los resultados estimados de la ecuación (1). Dado que solo podemos generar nuestras mediciones de la fluidez respecto a empleados asalariados formales ${ }^{21}$, los resultados que estudiamos se refieren a los mercados laborales formales de asalariados. La incapacidad de observar el mercado informal constituye una limitación de este estudio ${ }^{22}$. Sin embargo, supone un paso adelante en la literatura especializada porque, que nos conste, la relación entre la fluidez y el desempeño del mercado laboral no se había estudiado antes en el contexto de la región latinoamericana o en el de las economías en desarrollo en general. Centramos nuestra atención dos resultados en particular de los mercados laborales: por un lado, los trabajadores asalariados-formales como proporción de la totalidad de la fuerza de trabajo; por otro lado, los trabajadores asalariadosformales como proporción de la totalidad de la población en edad de trabajar. La primera variable es un componente de la tasa de empleo y se le denominará tasa de empleo formal. La segunda variable

${ }^{19}$ En el caso concreto que nos ocupa es poco probable que estos instrumentos demográficos mantengan una correlación con el error de medición de las mediciones de la fluidez, ya que las dos variables provienen de diferentes fuentes.

20 La estadística para la prueba de restricción sobreidentificadora se calcula como $N \times R_{u}^{2}$, donde $N$ y $R_{u}$ provienen de una regresión auxiliar de $\hat{\varepsilon}_{i t}$ sobre [XZ]. En esta regresión auxiliar, X representa la matriz de las covariantes exógenas y Z representa la matriz de instrumentos; $N \times R_{u}{ }^{2}$ se distribuye $\chi^{2}$ con grados de libertad equivalentes al número de restricciones sobreidentificadoras. La hipótesis nula de esta prueba es la exogeneidad de los instrumentos, matemáticamente, $H_{0}: E\left(Z^{\prime} u\right)=0$.

${ }^{21}$ Formales en el sentido de que trabajan en empresas que pagan impuestos a la nómina. No se trata esta de la última definición de informalidad de la Organización Internacional del Trabajo (OIT), pero es una definición institucionalista tradicional de la informalidad, y es la más atractiva dada la naturaleza de nuestros datos.

22 Este segmento del mercado es grande; además, parte de la creación de empleo observada en PILA puede ser el resultado de la formalización de puestos de trabajo que antes eran informales. 
es la tasa de ocupación formal-asalariada (trabajadores asalariados formales como proporción de la población en edad de trabajar).

En el cuadro 1 se muestra el resumen estadístico relativo a los controles, variables dependientes y mediciones de la fluidez. El período estudiado se caracterizó por una reducción del desempleo y por un importante crecimiento económico, por lo que no es sorprendente que la tasa promedio de contratación $(9,22 \%)$ sea más alta que la tasa promedio de desvinculación (8,47\%), o que la tasa promedio de creación de empleos $(5,57 \%)$ sea mayor que la tasa promedio de destrucción de empleos $(4,81 \%)$. A partir de estas cifras podemos caracterizar la relación promedio entre los flujos de trabajadores y de empleos. Como puede observarse, alrededor del $60 \%$ de la contratación correspondió a nuevos puestos de trabajo y aproximadamente el $57 \%$ de las desvinculaciones fueron por puestos de trabajo destruidos. Los datos a partir de los cuales calculamos nuestras mediciones de la fluidez contienen información acerca de más de 186.000 empresas con por lo menos dos empleados, lo que da un promedio total de casi 6,1 millones de trabajadores formales observados mensualmente a lo largo del período de estudio, lo que equivale al $57 \%$ de todo el empleo. En el anexo se presentan algunas características de las empresas. Por ejemplo, la empresa promedio tiene 33 empleados, de los cuales el 60\% son hombres y alrededor del 61\% ganan el salario mínimo; el $61 \%$ de las empresas son pequeñas, con menos de 20 empleados; y casi el 14\% de las empresas son medianas, con más de 20 pero menos de 100 empleados.

Cuadro 1

Resumen estadístico

\begin{tabular}{|c|c|c|c|c|c|}
\hline Variable & Observaciones & Media & $\begin{array}{l}\text { Desviación } \\
\text { estándar }\end{array}$ & Mínimo & Máximo \\
\hline $\begin{array}{l}\text { Trabajadores formales / población } \\
\text { económicamente activaa }\end{array}$ & 1725 & 0,48 & 0,08 & 0,27 & 0,59 \\
\hline $\begin{array}{l}\text { Trabajadores asalariados formales / } \\
\text { población económicamente activa }\end{array}$ & 1725 & 0,40 & 0,07 & 0,20 & 0,51 \\
\hline $\begin{array}{l}\text { Trabajadores asalariados formales } \\
\text { / fuerza de trabajo }{ }^{\mathrm{a}}\end{array}$ & 1725 & 35,13 & 6,66 & 17,28 & 45,51 \\
\hline $\begin{array}{l}\text { Trabajadores asalariados formales / } \\
\text { población en edad de trabajar }{ }^{\mathrm{a}}\end{array}$ & 1725 & 23,39 & 5,24 & 10,52 & 31,41 \\
\hline Tasa de contratación & 1725 & 9,22 & 2,57 & 3,02 & 24,47 \\
\hline Tasa de desvinculación & 1725 & 8,47 & 2,44 & 2,18 & 23,13 \\
\hline Tasa de creación de empleo & 1725 & 5,57 & 2,08 & 2,11 & 23,16 \\
\hline Tasa de destrucción de empleo & 1725 & 4,81 & 1,9 & 1,4 & 21,74 \\
\hline Tasa de reasignación de trabajadores & 1725 & 17,68 & 4,48 & 6,48 & 33,96 \\
\hline Tasa de reasignación de empleos & 1725 & 10,37 & 3,32 & 3,82 & 27,66 \\
\hline Tasa de rotación & 1725 & 7,31 & 2,59 & 0,67 & 15,98 \\
\hline Tasa de salario real por hora (en pesos) & 1725 & 4221 & 638 & 2771 & 8162 \\
\hline $\begin{array}{l}\text { Proporción de la población en edad de } \\
\text { trabajar con educación terciaria }\end{array}$ & 1725 & 0,25 & 0,06 & 0,13 & 0,42 \\
\hline Proporción de empresas del sector privado & 1725 & 0,97 & 0,02 & 0,88 & 0,99 \\
\hline Edad promedio de los empleados & 1725 & 37,41 & 0,81 & 35,1 & 39,61 \\
\hline Edad promedio de los empleados & 1725 & 1306 & 330 & 97 & 1569 \\
\hline Proporción de empleados hombres & 1725 & 0,63 & 0,03 & 0,55 & 0,69 \\
\hline $\begin{array}{l}\text { Demanda de mano de obra a } \\
\text { nivel de los departamentos }\end{array}$ & 1725 & 0,01 & 0,02 & $-0,05$ & 0,06 \\
\hline PIB per cápita & 1725 & 5675 & 188 & 5313 & 6152 \\
\hline $\begin{array}{l}\text { Costos de los empleadores (empresas) } \\
\text { (proporción de salarios medianos) }\end{array}$ & 1725 & 0,53 & 0,05 & 0,42 & 0,56 \\
\hline PIB trimestral con el filtro de Hodrick-Prescott & 1725 & -357 & 1112 & -2815 & 1599 \\
\hline Crecimiento del PIB departamental & 1725 & 4,31 & 4,58 & $-6,37$ & 25,18 \\
\hline
\end{tabular}

Fuente: Elaboración propia.

a Promedios ponderados que toman las contribuciones de cada área metropolitana al empleo total como ponderaciones. 
Durante el período objeto de estudio, la tasa de empleo asalariado formal alcanzó un promedio del 35\%, lo que representa la proporción de la fuerza de trabajo con un empleo asalariado formal. La tasa de ocupación de asalariado formal, o proporción de la población en edad de trabajar con un empleo asalariado formal, fue del $23,4 \%$. En su conjunto, la proporción de la fuerza de trabajo con un empleo formal (tasa de formalidad) ascendió al 48\%. El grupo más selecto de la población activa estaba compuesto por empleados con un puesto de trabajo asalariado formal, que representaban el $40 \%$ del total durante el período objeto de estudio. Cabe mencionar algunas otras características de la muestra de estimación de nuestra regresión. Por ejemplo, la tasa promedio de crecimiento departamental durante el período alcanzó el 4,31\%, la edad promedio de los empleados fue de 33,3 años y la tasa salarial promedio por hora en las áreas metropolitanas durante el período estudiado fue de 4.221 pesos colombianos a valores de 2008.

Analizamos tres mediciones de la fluidez, a saber, la WR, la JR y la CR, y dos resultados diferentes, la tasa de empleo asalariado formal y la tasa de ocupación asalariada formal. Estimamos cada una de las ecuaciones utilizando los mínimos cuadrados ordinarios (OLS) y la metodología de las variables instrumentales (véase el cuadro 2). En el caso de las variables instrumentales, utilizamos diversos instrumentos seleccionados a partir de los que se describen en la sección anterior. Para cada regresión elegimos la combinación de instrumentos que mantuviera la más firme correlación con las variables endógenas; además, llevamos a cabo pruebas de restricciones sobreidentificadoras con cada juego de instrumentos, y verificamos que no se rechazara la hipótesis de que el instrumento era válido. En general, los juegos de instrumentos utilizados son similares para todas las ecuaciones.

La unidad de observación en todos los modelos presentados es la de los mercados de trabajo, que definimos como áreas metropolitanas de Colombia. Pudimos recopilar toda la información necesaria para estimar la ecuación (1) para las 23 áreas metropolitanas colombianas, correspondiente al período transcurrido entre octubre de 2008 y diciembre de 2014. En general, las regresiones presentan un alto nivel de ajuste en todas las regresiones, alcanzando un R cuadrado superior al 90\%. Incluimos los efectos fijos del área metropolitana, los efectos fijos mensuales y los efectos fijos anuales en todas las regresiones. Todos estos efectos fijos explican una gran parte de la variación de nuestras variables dependientes. Las variables de control presentan signos coherentes y son significativas en la mayoría de las especificaciones que estimamos. Para todas nuestras variables dependientes, el PIB per cápita y la proporción de la población en edad de trabajar con educación terciaria presentan una correlación positiva y significativa con cada uno de estos índices laborales. Por lo que respecta a las tasas de empleo, el porcentaje de empresas privadas se correlaciona de manera negativa y significativa con este índice laboral. En otras palabras, en un mercado de trabajo en el que el sector público representa una proporción alta del conjunto del empleo, se evidencian mayores tasas de empleo. Para este mismo índice laboral, observamos una correlación significativa y positiva con la tasa de crecimiento real del PIB por departamento.

Tasa de reasignación de trabajadores (WR): La estimación de los mínimos cuadrados ordinarios muestran que la WR tiene un efecto positivo sobre el empleo asalariado formal y sobre las tasas de ocupación. Las magnitudes de esos efectos son bastante pequeñas: un aumento de un punto porcentual en la WR da lugar a un incremento de la tasa de empleo asalariado formal y de la tasa de ocupación asalariada formal de 0,030 y 0,029 puntos porcentuales, respectivamente. Con respecto a los resultados referentes a las variables instrumentales, encontramos una relación positiva y significativa entre la WR y los dos resultados. Las magnitudes de esas relaciones son sustancialmente más grandes que con los mínimos cuadrados ordinarios: un incremento de 1 punto porcentual en la WR aumenta las tasas de empleo asalariado formal y de ocupación en 0,26 y 0,24 puntos porcentuales, respectivamente. Estos resultados se ajustan a los obtenidos en Davis y Haltiwanger (2014) referente al mercado de trabajo de los Estados Unidos, lo que corrobora la posible influencia causal positiva de la WR sobre la tasa de empleo. 


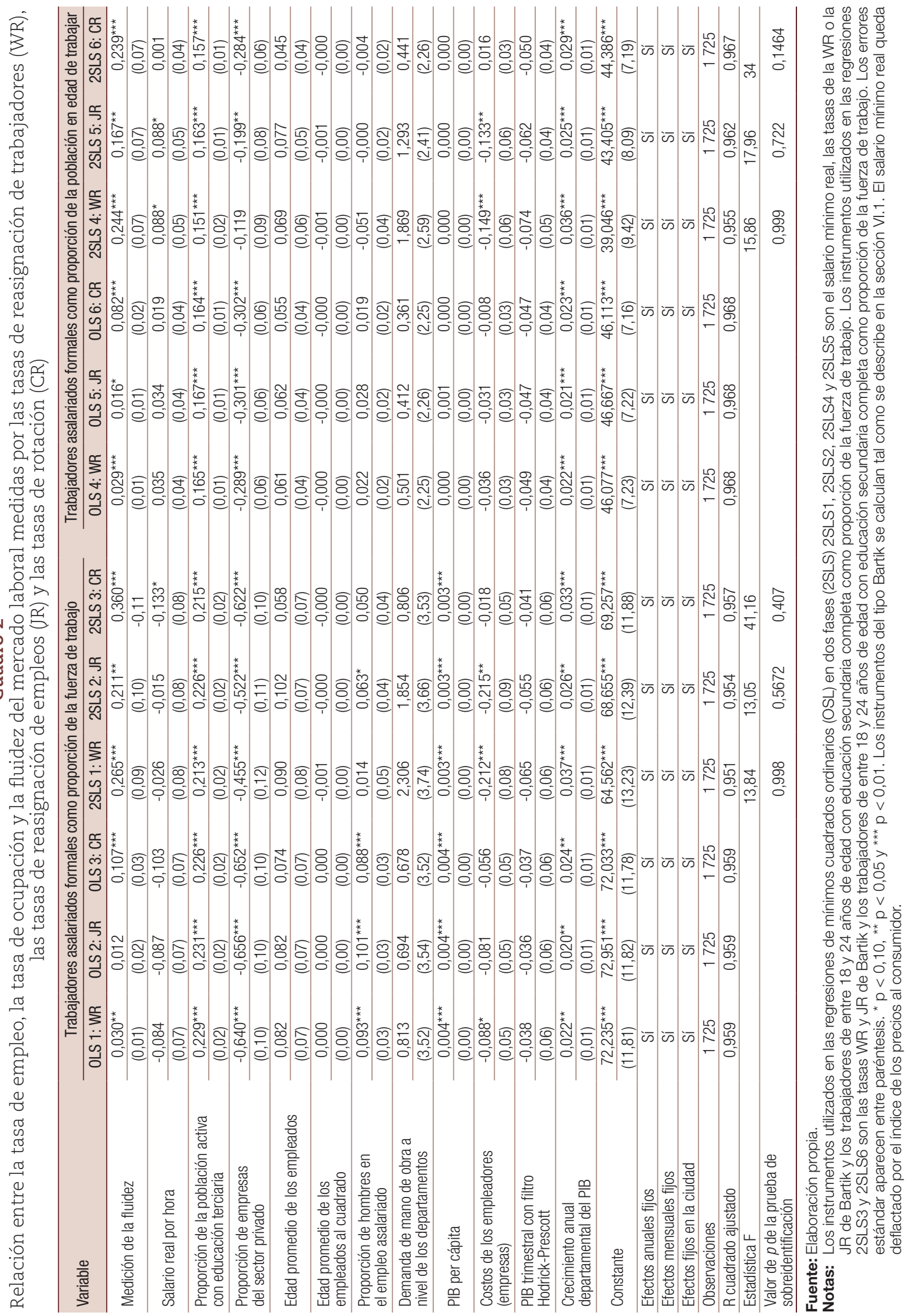


Tasa de reasignación de empleos (JR): Las estimaciones de los mínimos cuadrados ordinarios muestran un efecto positivo de la JR sobre las tasas de empleo asalariado formal y de ocupación, con magnitudes pequeñas en ambos casos y un efecto significativo solo en el caso de la tasa de ocupación asalariada formal. Por lo que se refiere a las variables instrumentales, encontramos que la JR tiene un posible efecto causal positivo y significativo sobre ambos resultados. Las magnitudes de esos efectos son mayores que las que se obtuvieron con la estimación de los mínimos cuadrados ordinarios. El efecto de un aumento de 1 punto porcentual consiste en un incremento de 0,21 y 0,17 puntos porcentuales en las tasas de empleo asalariado formal y de ocupación, respectivamente. Este resultado contrasta con las correlaciones negativas entre la JR y el crecimiento neto del empleo que se indica en Davis, Haltiwanger y Schuh (1996). Sin embargo, la evidencia de una JR contracíclica se sustenta en correlaciones incondicionales. Nuestros resultados son coherentes con los obtenidos por Davis y Haltiwanger (2014), quienes encuentran una posible influencia causal positiva de la JR sobre la tasa de empleo; al igual que surge de nuestras conclusiones, la JR tiene un efecto menor que la WR.

Tasa de rotación o churning (CR): Las estimaciones de los mínimos cuadrados ordinarios muestran que la CR guarda una relación positiva y significativa con ambos índices del mercado de trabajo. La magnitud de estas correlaciones es pequeña: sin embargo, es mayor que las estimadas utilizando los mínimos cuadrados ordinarios para los casos anteriores de la WR y la JR. Con un incremento de 1 punto porcentual en la CR, las tasas de empleo asalariado formal y de ocupación aumentan 0,11 y 0,08 puntos porcentuales, respectivamente. Por lo que respecta a las estimaciones de las variables instrumentales, como se indicó anteriormente, las magnitudes de los posibles efectos causales de la CR sobre nuestros resultados son sustancialmente mayores. Con un incremento de 1 punto porcentual en la CR, las tasas de empleo asalariado formal y de ocupación aumentan 0,36 y 0,24 puntos porcentuales, respectivamente.

Reseña general de los resultados: Tal como se explicó en la sección III, desde el punto de vista de la teoría del desempleo de equilibrio (Pissarides, 2000), sería de esperar que los incrementos en la WR y la CR dieran lugar a una mejora en el desempeño del mercado de trabajo. Por otra parte, a partir del marco teórico delineado en Shimer (2001) y Davis y Haltiwanger (2014), cabría suponer que los aumentos en todas las mediciones de la fluidez mejoraran nuestros dos índices del mercado laboral. Estos dos estudios proponen cauces teóricos por medio de los cuales la fluidez pueda generar mejoras en el desempeño del mercado laboral. De conformidad con Shimer (2001), las empresas encuentran más provechoso crear puestos de trabajo en mercados laborales más fluidos y compuestos por personas más jóvenes, y en los que resulta más económico contratar a nuevos empleados. Según Davis y Haltiwanger (2014), los trabajadores reciben ofertas de empleo más frecuentes en mercados de trabajo fluidos, en los que los períodos de desempleo son más cortos, lo que previene así una costosa pérdida de capital humano. Dichos cauces justifican también los efectos positivos de la WR, la CR y la JR sobre el desempeño de los mercados laborales (Davis y Haltiwanger, 2014).

Nuestras estimaciones de los mínimos cuadrados ordinarios muestran una correlación positiva y casi siempre significativa entre todas las mediciones de la fluidez y los índices de los mercados laborales. Las estimaciones de las variables instrumentales ponen en evidencia un posible efecto causal significativo respecto a todas las mediciones de la fluidez sobre los índices del mercado laboral y uno que es sustancialmente mayor al que dan a entender las regresiones de los mínimos cuadrados ordinarios. Se subestiman entonces los coeficientes de los mínimos cuadrados ordinarios, ya que los factores que varían a lo largo del tiempo presentan una correlación negativa con las mediciones de la 
fluidez. Los resultados que obtuvimos se muestran robustos en lo referente a las modificaciones en el conjunto de instrumentos y cambios en la muestra considerada ${ }^{23}$.

Las magnitudes de los efectos que tienen la WR, la JR y la CR sobre los índices laborales son bastante similares; sin embargo, la JR tiene un efecto menor sobre el empleo asalariado formal y la ocupación que la WR o la CR. La CR tiene el mayor efecto sobre el empleo. Estos patrones se ajustan al marco teórico de la teoría del desempleo de equilibrio, que hace hincapié en el papel de los flujos de trabajadores (especialmente las transiciones de empleo a empleo) sobre la reducción de la tasa de desempleo de equilibrio. En una versión general de estos modelos de búsqueda, el mayor número de flujos de empleos dinámicos responde al incremento de la dinámica del flujo de trabajadores a raíz de las reducciones en los costos de la búsqueda en el trabajo. En concreto, un determinado incremento en los flujos de trabajadores (resultante de las transiciones de empleo a empleo) dará lugar a un aumento de la creación de puestos de trabajo y a una reducción de su destrucción; por ende, el incremento en los flujos de trabajo derivados de la mayor creación de puestos de trabajo se verá parcialmente contrarrestado por una menor destrucción de empleos. Por lo tanto, teóricamente, el efecto de los flujos de empleos en el desempleo debería ser menor porque los mayores flujos de empleo resultantes de un incremento dado de los flujos de trabajadores se ven parcialmente contrarrestados por una menor destrucción de empleos. Si bien se espera que la JR tenga un efecto menor que la WR, los modelos de búsqueda predicen efectos positivos para la JR a la hora de reducir la tasa de desempleo de equilibrio, por la sencilla razón de que los flujos de trabajo son un componente de los flujos de trabajadores.

El mercado de trabajo urbano informal en Colombia presenta un tamaño considerable. Sin embargo, en 2016 los trabajadores formales constituían casi el 51\% de la población urbana activa ${ }^{24}$, mientras que los trabajadores asalariados formales eran más del $43 \%$ de la población activa urbana. Con los datos de que disponemos, no es posible calcular las mediciones de fluidez para los trabajadores informales. Es probable que en el mercado de trabajo formal tenga lugar el mayor grado de dinamismo de los trabajadores y los empleos, dado que los empleos y los trabajadores informales corresponden a las pequeñas empresas o son personas que trabajan por cuenta propia ${ }^{25}$. Sin embargo, existe evidencia referente a economías similares en la región según la cual los trabajadores por cuenta propia presentan elevados índices de transición anual, especialmente desde el estatus de trabajador por cuenta propia al de empleado (Gluzmann, Jaume y Gasparini, 2012); estas dinámicas solo se pueden captar parcialmente por nuestras mediciones de la fluidez, por ejemplo por medio de la creación de empleos formales. Ello constituye una limitación de este estudio ya que, tal como se mencionó anteriormente, solo podemos calcular las mediciones de la fluidez correspondientes al mercado de trabajo formal. Aunque no observamos flujos de mercado de trabajo informal, los indicios apuntan en la dirección de que las dinámicas de los trabajadores y empleos informales se vinculan mayormente a la creación de puestos de trabajo y a la contratación en el mercado formal, ya que la

${ }^{23}$ Como prueba de robustez, estimamos todas las regresiones de variables instrumentales, excluyéndose el salario mínimo del conjunto de instrumentos. Para el empleo asalariado formal, el aumento de 1 punto porcentual en la WR, la JR y la CR aumenta esta tasa en 0,271, 0,188 y 0,360 puntos porcentuales respectivamente. Respecto a la tasa de ocupación asalariada formal, un incremento de punto porcentual en la WR, la JR y la CR aumenta esta tasa en 0,24, 0,10 y 0,24 puntos porcentuales respectivamente. En todos los casos, los coeficientes son significativos desde el punto de vista estadístico. Como prueba de robustez adicional, el punto de partida de la muestra utilizada para todas las regresiones se desplazó hacia atrás y hacia adelante uno y dos meses, sin cambios importantes en los resultados.

${ }^{24}$ Esta tasa se calcula utilizando la definición de informalidad de la Organización Mundial del Trabajo (OIT), que abarca a los trabajadores no profesionales informales en empresas con cinco empleados o menos.

${ }^{25}$ La proporción de toda la fluidez derivada de las empresas muy pequeñas es ínfima. Las empresas con dos o cinco empleados representan solo el 1,09\% del total de flujos de empleos, el 5,87\% del total de flujos de trabajadores, y 3,57\% de los flujos de rotación. 
reducción de la tasa de informalidad en Colombia que se ha observado en los últimos años se explica en parte por la formalización de las empresas y los trabajos como resultado de políticas favorables en materia de mercado de trabajo (Morales y Medina, 2017; Fernández y Villar, 2017). En resumen, aunque no podemos observar las dinámicas de empleos y trabajadores en el caso de los trabajadores informales, consideramos que estamos en condiciones de realizar un aporte significativo acerca de una parte importante del mercado de trabajo.

En un artículo reciente, Flórez y otros (2018) compararon las mediciones de la fluidez de los Estados Unidos y Colombia. El análisis de estos autores revela diferencias entre los dos mercados de trabajo: por ejemplo, llegan a la conclusión de que la tasa trimestral de rotación es casi dos veces más grande en los Estados Unidos que en Colombia ${ }^{26}$. Esta comparación ha de abordarse con cautela, en vista de las diferencias en la composición de los mercados formales e informales de las dos economías y el hecho de que las mediciones de los flujos de trabajadores en los Estados Unidos cambian en función de la fuente (Flórez y otros, 2017). Sin embargo, dadas las características de los dos mercados de trabajo, existen muchas razones para suponer que el de los Estados Unidos sea más fluido que el de Colombia. La literatura anterior muestra que la protección del empleo y la existencia de instituciones de un mercado laboral rígido desempeñan un importante papel a la hora de reducir el dinamismo del mercado de trabajo (Blanchard y Portugal, 2010). Colombia tiene niveles altos de protección del empleo, con 4,5 semanas de licencia por maternidad y una serie de leyes que rigen el despido improcedente y los costos del despido ${ }^{27}$ (Cardona-Sosa y otros, 2018; Cardona, Flórez y Morales, 2016). Además, antes de la reforma tributaria de 2013, Colombia tenía uno de los niveles más altos de contribuciones de nómina en la región latinoamericana, en la que las contribuciones de nómina de empleadores y empleados ascendían al $60 \%$ de los salarios. Algunas de estas instituciones podrían agregar rigidez al mercado.

Las políticas recientes probablemente han contribuido a aumentar la fluidez y, tal como se indica en la sección $\mathrm{V}$, el mercado de trabajo pasó a ser sustancialmente más dinámico después de 2010. Entre las políticas que se han venido implementando últimamente cabe mencionar una importante reducción de las contribuciones de nómina en 2013, así como una serie de subsidios a dichas contribuciones para los empleados jóvenes y las nuevas empresas formales (ley del primer empleo, implementada en 2010). Existen evidencias de que estas políticas han contribuido a impulsar el empleo formal (Morales y Medina, 2017; Fernández y Villar, 2017).

Por último, aportamos evidencia de una posible relación causal entre fluidez y mejor desempeño del mercado de trabajo en términos de empleo y ocupación. Sin embargo, esta evidencia ha de abordarse con cautela, ya que solo analizamos dos de los muchos posibles resultados que podrían verse influenciados por la fluidez del mercado de trabajo. Tal como se indica en la sección III, en la literatura especializada se señalan aspectos no deseados de la fluidez del mercado de trabajo, muchos de ellos relativos a la inestabilidad laboral. La evidencia que presenta la literatura muestra que es probable que la inestabilidad laboral sea algo negativo para los trabajadores y para las empresas, dándose a entender que podría haber una relación positiva entre la antigüedad laboral y la productividad de una empresa (Auer, Berg y Coulibaly, 2005); se prevé que los mayores flujos de trabajadores y de empleos en la economía reducirán la permanencia promedio en el empleo.

\footnotetext{
26 Llegan a esa conclusión utilizando tasas de rotación trimestrales calculadas con base en la PILA en el caso de Colombia, mientras que en el caso de los Estados Unidos utilizan la tasa de rotación trimestral obtenida por la Oficina de Censos a partir de la encuesta de la dinámica longitudinal de empleadores y hogares. El período de comparación abarcó de 2009 a 2016.

27 Los despidos injustificados son caros en Colombia: las empresas tienen que pagar salarios correspondientes a 20 días por cada año trabajado. Se trata de una política similar a la de Portugal, uno de los países que tiene costos de despido más altos. En 2015, Colombia ocupaba el número 29 en el índice de la Organización para la Cooperación y el Desarrollo Económico de protección de los trabajadores permanentes, mientras que Portugal estaba en el número 13 y los Estados Unidos en el 69 de un total de 71 países respecto a los cuales se podía calcular el índice.
} 


\section{Conclusiones}

En este artículo se analizan la fluidez del mercado de trabajo formal en Colombia y la relación entre las mediciones de la fluidez y algunos resultados del mercado de trabajo formal, en particular las tasas de empleo y de ocupación correspondientes a los trabajadores asalariados formales. Los trabajos empíricos llevados a cabo recientemente y que se centran en la economía de los Estados Unidos dan a entender que la fluidez ejerce una influencia positiva sobre el desempeño del mercado laboral (Davis y Haltiwanger, 2014; Molloy y otros, 2016; Shimer, 2001). Además, las elaboraciones teóricas recientes (modelos de desempleo de equilibrio) predicen que los factores que reducen los costos de búsqueda y, por ende, aumentan la fluidez, darán lugar a una caída de la tasa de equilibrio del desempleo. Nuestro trabajo contribuye a la literatura empírica al estimar los efectos de la fluidez sobre el desempeño del mercado de trabajo formal utilizando información relativa a todos los sectores económicos, no solo las manufacturas. Por otra parte, no nos consta que exista ningún otro estudio sobre el impacto de la fluidez sobre el desempeño del mercado de trabajo en una economía en desarrollo.

Analizamos las mediciones estándar de la fluidez correspondientes al período transcurrido entre octubre de 2008 y diciembre de 2014. Detectamos una sólida tendencia alcista a partir de 2009 en las tres mediciones de fluidez que fueron objeto de estudio en este artículo. Se trata de un período en el cual el desempleo experimentó una pronunciada caída. Gracias a la utilización de modelos econométricos, aportamos evidencias de que las mediciones de la fluidez ejercen un impacto causal plausible, consistente y pronunciado sobre ambos resultados del mercado de trabajo. Utilizando modelos sencillos de mínimos cuadrados ordinarios, llegamos a la conclusión de que casi todas las mediciones de la fluidez mantienen una correlación positiva y significativa con las tasas de empleo asalariado formal y de ocupación; sin embargo, las magnitudes de esas correlaciones son pequeñas. Cuando se sigue un enfoque de regresión de variable instrumental, se advierte que todas las mediciones de la fluidez tienen un efecto positivo y significativo sobre los resultados del mercado de trabajo, y las magnitudes de los coeficientes son sustancialmente más altas.

Llegamos a la conclusión de que un incremento en todas nuestras mediciones de la fluidez da lugar a un aumento de las tasas de empleo asalariado formal y de ocupación. Las magnitudes del efecto son relativamente similares para cada resultado. Un incremento de 1 punto porcentual en cualquiera de las mediciones de la fluidez provoca un aumento en las tasas de empleo formal de entre 0,21 y 0,36 puntos porcentuales en dichas tasas, mientras que un alza de 1 punto porcentual en la JR, la WR o la CR da lugar a un incremento de las tasas de ocupación formal de entre 0,17 y 0,24 puntos porcentuales. La JR es la variable con el menor impacto, mientras que a la CR corresponde el mayor impacto en lo referente a las tasas de empleo: ello se ajusta a las predicciones teóricas para el desempleo de equilibrio.

Según nuestras conclusiones, la existencia de mercados de trabajo más fluidos ha repercutido favorablemente en el mercado de trabajo formal de Colombia en los últimos años: el buen desempeño del mercado de trabajo observado entre 2009 y 2014 obedece al menos parcialmente al mayor grado de fluidez. La literatura especializada sobre los flujos laborales suele indicar una relación positiva entre la fluidez y la flexibilidad del mercado laboral (Davis y Haltiwanger, 2014; Molloy y otros, 2016). La evidencia que presentamos en este artículo arroja luz sobre los posibles efectos beneficiosos de las políticas que buscan una mayor flexibilidad. Nuestras conclusiones son pertinentes para una comprensión más amplia del mercado de trabajo formal en los países en desarrollo; aportamos evidencias de que las políticas que buscan una mayor fluidez y flexibilidad son susceptibles de impulsar el empleo formal. Sin embargo, esta afirmación ha de abordarse con cautela, teniendo en cuenta la anterior literatura sobre los efectos de las políticas laborales en los países en desarrollo. La conclusión de un metaanálisis del tema presentada en un informe del Banco Mundial en 2012 (Banco Mundial, 2012) es que tanto una regulación excesiva de los mercados de trabajo como una regulación 
insuficiente de ellos reducen la productividad de las empresas y la creación de puestos de trabajo. En el informe se sostiene que los efectos negativos de la mayoría de las regulaciones laborales estándar son sutiles o desconocidas.

Nuestras conclusiones son pertinentes para el análisis de los actuales mercados de trabajo en la región latinoamericana y por ende en otros países en desarrollo en que se han implementado en fecha reciente cambios en las políticas laborales. Se prevé que algunos de esos cambios normativos darán lugar a una mayor flexibilidad. En México, por ejemplo, en el marco de la reforma de la política laboral implementada en 2012 se introdujeron contratos de trabajo innovadores y más flexibles. En Colombia, en 2012 la reforma tributaria redujo las contribuciones de nómina, lo que ha promovido la creación de empleos formales (Morales y Medina, 2016). En el Ecuador, la reforma laboral implementada en 2016 introdujo nuevos contratos subsidiados para los trabajadores jóvenes, así como una concepción más flexible de los turnos de trabajo y de la licencia no retribuida para el cuidado de los hijos a fin de complementar la licencia por maternidad normal. Se prevé que algunas otras reformas laborales, como la de Chile - que concede derechos a los sindicatos - reducirán la flexibilidad. En este artículo identificamos una posible relación causal entre la fluidez y el desempeño de trabajo formal, que puede utilizarse como criterio para predecir las posibles consecuencias de todas estas políticas puestas en práctica en las economías en desarrollo. No hay mucho que podamos decir acerca de los mercados de trabajo informales, y ello constituye una limitación de este estudio, teniendo en cuenta el tamaño considerable del sector informal en la economía colombiana. Hace falta un mayor trabajo de investigación sobre las mediciones indirectas (proxy) de los flujos de mano de obra para el mercado informal; no nos consta que exista ni un solo estudio que permita analizar la fluidez del mercado de trabajo del sector informal de algún país. Este desconocimiento de la dinámica del mercado de trabajo se debe a la imposibilidad de observar el mercado informal a través de las fuentes de datos tradicionales.

\section{Bibliografía}

Akerlof, G. A. y otros (1988), "Job switching and job satisfaction in the U.S. labor market”, Brookings Papers on Economic Activity, № 2, Washington, D.C., The Brookings Institution.

Auer, P., J. Berg e I. Coulibaly (2005), “¿El trabajo estable mejora la productividad?”, Revista Internacional del Trabajo, vol. 124, № 3, Ginebra, Organización Internacional del Trabajo (OIT).

Autor, D. H., J. J. Donohue III y S. J. Schwab (2006), "The costs of wrongful-discharge laws", The Review of Economics and Statistics, vol. 88, № 2, Cambridge, The MIT Press.

Autor, D. H. y M. G. Duggan (2003), "The rise in the disability rolls and the decline in unemployment", The Quarterly Journal of Economics, vol. 118, N 1, Oxford, Oxford University Press.

Autor, D. H., W. R. Kerr y A. D. Kugler (2007), "Does employment protection reduce productivity? Evidence form US states", The Economic Journal, vol. 117, № 521, Wiley.

Banco Mundial (2012), World Development Report 2013: Jobs, Washington, D.C.

Bartik, T. J. (1991), Who Benefits from State and Local Economic Development Policies?, Kalamazoo, Upjohn Press.

Bjelland, M. y otros (2011), "Employer-to-employer flows in the United States: estimates using linked employeremployee data", Journal of Business \& Economic Statistics, vol. 29, № 4, Abingdon, Taylor \& Francis.

Blanchard, O. y L. Katz (1992), "Regional evolutions", Brookings Papers on Economic Activity, № 1, Washington, D.C., The Brookings Institution.

Blanchard, O. y P. Portugal (2001), "What hides behind an unemployment rate: comparing Portuguese and U.S. labor markets", The American Economic Review, vol. 91, № 1, Nashville, American Economic Association.

Boeri, T. y J. F. Jimeno (2005), "The effects of employment protection: learning from variable enforcement", European Economic Review, vol. 49, № 8, Amsterdam, Elsevier.

Bound, J. y H. J. Holzer (2000), "Demand shifts, population adjustments, and labor market outcomes during the 1980s", Journal of Labor Economics, vol. 18, № 1, Chicago, The University of Chicago Press. 
Burgess, S., J. Lane y D. Stevens (2000), "Job flows, worker flows, and churning”, Journal of Labor Economics, vol. 18, No 3, Chicago, The University of Chicago Press.

Cardona-Sosa, L. y otros (2018), "How does the Household Labour Supply Respond to the Unemployment of the Household Head?", LABOUR, vol. 32, № 4.

Cardona, L., L. A. Flórez y L. Morales (2016), "Intra-household labour supply after an unemployment event: the added worker effect”, Borradores de Economía, № 944, Bogotá, Banco de la República.

Davis, S. J., J. Faberman y J. C. Haltiwanger (2012), "Labor market flows in the cross section and over time", Journal of Monetary Economics, vol. 59, $\mathrm{N}^{\circ} 1$, Amsterdam, Elsevier.

Davis, S. J. y J. Haltiwanger (2014), "Labor market fluidity and economic performance", NBER Working Paper, № 20479, Cambridge, Oficina Nacional de Investigaciones Económicas.

(1999), "Gross job flows", Handbook of Labor Economics, O. Ashenfelter y D. Card (eds.), vol. 3, Amsterdam, Elsevier.

(1992), "Gross job creation, gross job destruction, and employment reallocation", The Quarterly Journal of Economics, vol. 107, No 3, Oxford, Oxford University Press.

Davis, S. J., J. Haltiwanger y S. Schuh (1996), Job Creation and Destruction, Cambridge, The MIT Press.

Davis, S. J. y T. M. Von Wachter (2011), "Recessions and the cost of job loss", NBER Working Paper, № 17638, Cambridge, Oficina Nacional de Investigaciones Económicas.

Decker, R. y otros (2014), "The role of entrepreneurship in US job creation and economic dynamism", The Journal of Economic Perspectives, vol. 28, N 3, Nashville, American Economic Association.

Fernández, C. y L. Villar (2017), "The impact of lowering the payroll tax on informality in Colombia", Economía, vol. 18, N 1, Washington, D.C., Brookings Institution Press.

Flórez, L. A. y otros (2018), "Labour flows across firm's size, economic sectors and wages: evidence from employer-employee linked panel”, Borradores de Economía, № 1013, Bogotá, Banco de la República.

Gluzmann, P., D. Jaume y L. Gasparini (2012), "Decisiones laborales en América Latina: el caso de los emprendedores. Un estudio sobre la base de encuestas de hogares", Documento de Trabajo, № 137, Centro de Estudios Distributivos, Laborales y Sociales (CEDLAS).

Gómez-Salvador, R., J. Messina y G. Vallanti (2004), "Gross job flows and institutions in Europe", Labour Economics, vol. 11, № 4, Amsterdam, Elsevier.

Hyatt, H. R. y J. R. Spletzer (2013), "The recent decline in employment dynamics", IZA Journal of Labor Economics, vol. 2, № 1, Springer.

Melo, L. y C. Ballesteros (2014), "Impacto de los factores externos sobre la creación y destrucción de empleo en el sector manufacturero colombiano", Lecturas de Economía, № 81, Medellín, Universidad de Antioquia. (2013), "Creación, destrucción y reasignación del empleo en el sector manufacturero", Revista de Economía Institucional, vol. 15, № 28, Bogotá, Universidad Externado de Colombia.

Molloy, R. y otros (2016), "Understanding declining fluidity in the US labor market", Brookings Papers on Economic Activity, Washington, D.C., The Brookings Institution.

Morales, L. F., y D. Medina (2016), "Labor fluidity and performance of labor outcomes in Colombia: evidence from employer-employee linked panel”, Borradores de Economía, No. 926, Bogotá, Banco de la República.

Morales, L. F. y C. Medina (2017), "Assessing the effect of payroll taxes on formal employment: the case of the 2012 tax reform in Colombia", Economía, vol. 18, № 1, Washington, D.C., Brookings Institution Press. (2016), "Assessing the effect of payroll taxes on formal employment: the case of the 2012 tax reform in Colombia”, Borradores de Economía, № 971, Bogotá, Banco de la República.

Pagés, C., G. Pierre y S. Scarpetta (2009), Job Creation in Latin America and the Caribbean. Recent Trends and Policy Challenges, Washington, D.C., Banco Mundial/Palgrave Macmillan.

Pissarides, C. (2000), Equilibrium Unemployment Theory, Cambridge, The MIT Press.

Shimer, R. (2001), "The impact of young workers on the aggregate labor market", The Quarterly Journal of Economics, vol. 116, № 3, Oxford, Oxford University Press. 


\section{Anexo A1}

Cuadro A1.1

Resumen estadístico referente a las empresas

\begin{tabular}{lccc}
\hline Variable & Observaciones & Media & $\begin{array}{c}\text { Desviación } \\
\text { estándar }\end{array}$ \\
\hline Empleo & 13977725 & 32,65387 & 263,3392 \\
\hline Empresas privadas & 13977725 & 0,972648 & 0,163108 \\
\hline Salarios promedio como proporción del salario mínimo & 13958019 & 1,554001 & 1,199409 \\
\hline Proporción de salarios menores o iguales al salario mínimo & 13977725 & 60,98038 & 39,64137 \\
\hline Proporción de hombres & 13977725 & 59,72614 & 31,84601 \\
\hline Proporción de empresas con 20 empleados o menos & 13977725 & 0,815355 & 0,3880093 \\
\hline Proporción de empresas con más de 20 pero menos de 100 empleados & 13977725 & 0,138829 & 0,3457676 \\
\hline Proporción de empresas con más de 100 empleados & 13977725 & 0,045816 & 0,2090867 \\
\hline
\end{tabular}

Fuente: Elaboración propia. 